\title{
RELACIÓN ENTRE VALOR DE LA EMPRESA, DIVERSIFICACIÓN Y GOBIERNO CORPORATIVO
}

\section{Carlos Maquieria V.*}

Escuela de Negocios

Universidad de Chile

Christian Espinoza

\author{
Escuela de Negocios \\ Universidad de Chile
}

(Recibido 24 de julio 2005, aceptado 31 de agosto 2005)

\section{Resumen}

Este artículo analiza la relación existente entre el desempeño de las empresas chilenas, la diversificación de sus negocios, el grado de concentración de la propiedad y el tipo de controlador. Se trabaja con un panel balanceado de 52 sociedades anónimas que transaron sus acciones durante el período 1995-2002. En cuanto a la diversificación y desempeño no es posible encontrar una relación que sea concluyente sobre la base de las hipótesis propuestas en la literatura. Esto podría deberse a que los beneficios que se derivan de la diversificación serían similares a los costos de tal estrategia. En relación con la propiedad del controlador, cuando ésta es mayor al $40 \%$, se observa una relación positiva y significativa con el número de negocios, Herfindahl y Entropía, concordando con la hipótesis de alineación de intereses. También se encuentra una débil evidencia a favor de la postura de que los flujos internos de la empresa constituyen un instrumento que permite expropiar riqueza a los minoritarios, antes que reflejar un mercado de capital interno eficiente. En cuanto a la relación que tiene la concentración de la propiedad y el desempeño de la empresa, los resultados de mayor relevancia son alcanzados cuando se hace la distinción por tipo de controlador. Para el caso de las familias, se observa una relación positiva entre desempeño y control hasta un $40 \%$ de propiedad del controlador, para luego mostrar una relación negativa a partir de un $65 \%$ de propiedad. Para el caso de los ejecutivos, la evidencia muestra que su participación en la propiedad no tiene una relación significativa con el desempeño hasta que llega a un $65 \%$, punto a partir del cual se relaciona positivamente con el desempeño de la empresa. Por último, en las multinacionales no es posible distinguir ningún patrón de comportamiento que sea significativo.

\section{Abstract}

This paper studies the relation between performance, diversification and ownership concentration for Chilean companies. We use balanced panel data for 52 public companies in 1995-2002 time period. We do not find conclusive results for the relation between performance and diversification. When the major owner has more than 40 percent of the company stocks we find a positive relation between this and the number of line of business in which the firm is

* Escuela de Negocios, Universidad de Chile. Teléfono: (562) 678-3375. Correo Electrónico: cmaquieira@facea.uchile.cl 
involved. This last result is consistent with the alignment incentive hypothesis. Analyzing internal cash flows, we find weak evidence for the wealth redistribution hypothesis between major stockholders and minorities. When the major stockholder belongs to the family we observe a positive relation between performance and ownership if the major stockholder has more than 40 percent of the shares. Once the major owner has more than 65 percent of the stocks this relation is negative. In the case of executives we find a positive relation between ownership and performance if they have more than 65 percent of the stocks. Finally, we do not report any significant relation when the major stockholder is a multinational company.

\section{Clasificación JEL: M14, M21}

Palabras clave: Hipótesis de eficiencia de mercado de capitales, Hipótesis de costos de agencia, Hipótesis de estructura de propiedad, Hipótesis de grupos económicos, Hipótesis de rendimiento y diversificación

\section{Introducción}

Este artículo analiza la relación existente entre el desempeño de las empresas productivas chilenas, la diversificación de sus negocios, el grado de concentración de la propiedad y el tipo de controlador. Con este propósito se obtiene información de la Superintendencia de Valores y Seguros (SVS) y de la Bolsa de Comercio de Santiago y se construye un panel balanceado de 52 sociedades anónimas que transaron sus acciones durante el período 1995 - 2002.

Con respecto a la diversificación, la hipótesis de eficiencia de mercado de capitales internos (Tarziján, 1999) postula que tal estrategia responde a la imposibilidad de la firma de dar una señal creíble al mercado sobre su política de inversión, lo que lleva a que opere bajo restricciones de capital externo con un alto costo en la obtención de fondos. Esto genera incentivos para que la administración desarrolle un mercado de capitales interno que permita asignar eficientemente los recursos entre las unidades de negocios, movilizando fondos desde aquellas unidades con excedentes de caja hacia las que presentan oportunidades de crecimiento, pero que no tienen suficientes recursos para financiarlas. No obstante, esto podría elevar los potenciales costos de agencia asociados a la diversificación, ya que permitiría a los insiders y accionistas controladores conducir las empresas en función de sus propios intereses, como también expropiar riqueza a los accionistas minoritarios (Lins y Servaes, 1999), lo cual tomaría mayor relevancia ante la existencia de una débil ley de protección a minoritarios (La Porta et al., 1997), bajos estándares contables e instituciones con baja capacidad de fiscalización.

Otra teoría a favor de la diversificación es la hipótesis de coaseguro de la deuda. Ésta argumenta que la diversificación permite elevar la capacidad de endeudamiento de la firma al reducir su riesgo específico y estabilizar sus flujos, como resultado de expandir sus negocios a segmentos con ingresos que presentan una baja correlación entre sí. Al respecto, Mansi y Reeb (2002) -bajo el supuesto de que la diversificación corporativa reduce el riesgo de la empresa- plantean que tal estrategia va a impactar negativamente el valor de los accionistas, pero fortalecerá los derechos de los bonistas, no alterándose en forma significativa el valor de la firma, dependiendo el impacto de la estructura de deuda de la compañía.

Por otra parte, están las teorías donde la diversificación se relaciona negativamente con el valor de la firma debido a los costos de agencia. Para este 
estudio se han identificado dos conflictos de agencia con origen en el accionista controlador: (1) su interés por transformar la empresa en un imperio personal de carácter único y (2) la necesidad de diversificar el riesgo de su inversión personal en la empresa. En el primer caso, el accionista controlador destina a su discreción recursos en consumos no pecuniarios e inversiones en proyectos de baja rentabilidad, siendo la diversificación un medio para el crecimiento de la empresa y su transformación a un carácter único (Jensen, 1986). El segundo caso (hipótesis de portafolio) se basa en la aversión al riesgo del accionista controlador quien, si percibe que su riqueza está estrechamente ligada al destino de la empresa, tendrá incentivos para adoptar estrategias de diversificación con el fin de reducir el riesgo de su portafolio personal, aún cuando ello resulte perjudicial para el resto de los accionistas (Amihud \& Lev, 1981).

Lins \& Servaes (1999) y Khanna \& Palepu (1999) hacen énfasis en que los beneficios y costos derivados de la diversificación, su importancia se relaciona con el grado en que la empresa esté inmersa en un mercado desarrollado o en una economía emergente. Para el primero, las empresas diversificadas tienen un menor desempeño que sus contrapartes focalizadas, identificándose como motivos principales la inapropiada asignación de responsabilidades, la ineficiente distribución de recursos con subsidios cruzados entre las divisiones (Berger \& Ofek, 1995), el pobre gobierno corporativo y los conflictos de poder interno. Por el contrario, según Khanna \& Palepu (1997, 1999), las firmas diversificadas en mercados emergentes pueden imitar las funciones benéficas de varias instituciones que están presentes en los mercados desarrollados, pudiendo así enfrentar de mejor forma que las empresas focalizadas las restricciones de capital, el cumplimiento de contratos y relaciones laborales, entre otras. Existe, además, otra línea de estudio donde la diversificación nace como respuesta al pobre desempeño de la empresa, la cual ya estaba sujeta a un descuento antes de diversificar (Lang \& Stulz, 1994) e incluso ser el resultado de un proceso endógeno (Villalonga, 2002).

En cuanto a la concentración de la propiedad, cuando un accionista-administrador puede ejercer el control sobre la empresa con una participación relativamente baja, y el resto de la propiedad accionaria se encuentra demasiado dispersa como para tener incentivos a monitorear, se debería esperar un mayor respaldo a la hipótesis de costos de agencia, que dice relación con la acción de insiders tendientes a satisfacer sus intereses personales en forma de consumo no pecuniario y objetivos de negocios personales. Por otra parte, en la medida que el controlador eleva su participación en la firma -y en línea con lo que dicta la hipótesis de convergencia de intereses (Jensen \& Meckling, 1976)-, se espera que los insiders pongan mayor control en el desempeño de la empresa, por el impacto que ello significa en su riqueza. Como señalan Shleifer \& Vishny (1986), los grandes accionistas internalizan en mayor grado las externalidades positivas de proveer servicios de monitoreo, reduciendo así los problemas de agencia. Esta hipótesis sugiere una relación uniformemente positiva entre el valor de la empresa y la propiedad de los insiders-controladores, a cualquier nivel de participación. Por último, a partir de cierto nivel de propiedad se podría esperar un mayor grado de asimetría de información entre los insiders y los outsiders. Así, queda abierta la posibilidad de que los controladores de la empresa incurran en prácticas conducentes a expropiar riqueza desde los minoritarios (hipótesis de expropiación). Como exponen Maquieira et al. (2003), 
la existencia de un inversionista controlador, o un pacto formal o tácito entre los principales accionistas que les permita ejercer el control, se traducirá en un descuento sobre el valor de las acciones de las sociedades de inversión, como reflejo del riesgo de expropiación. A lo anterior se debe agregar que la normativa legal en Chile tiene su base en el código francés, al que La Porta et al. (1997) distingue como el de menor protección a los minoritarios en relación a un sistema de ley común. Cabe señalar que para Chile en gran parte del período 1995-2002 no había una normativa que regulara los cambios de control en las sociedades anónimas abiertas. ${ }^{1}$ Sin embargo, esto cambió con la entrada en vigencia -el 20 de diciembre de 2000- de la Ley 19.705, que vino a regular las ofertas públicas de acciones (OPA) y estableció los gobiernos corporativos. No obstante, en dicha ley también se incorporó el artículo $10^{\circ}$ transitorio, al cual una sociedad podía acogerse voluntariamente para que su controlador no quedara sujeto a la nueva normativa y así pudiese enajenar libremente su participación en la sociedad por un plazo de hasta tres años. En función de lo anterior, se podría esperar que una mayor concentración de propiedad estuviese asociada con un mayor descuento en el valor de la empresa, debido al riesgo de expropiación ${ }^{2}$ y al cobro de un premio por liquidez al disminuir las acciones que se transan en el mercado abierto. Por otra parte, también se plantea que la relación entre concentración de la propiedad y desempeño de la empresa es el resultado de un proceso endógeno, donde ninguna relación sistemática debiera observarse entre tales variables (Demsetz \& Villalonga, 2001).

En relación a la identidad del accionista controlador, dependiendo de su naturaleza podrían darse distintos efectos e incentivos para un mismo nivel de participación en la propiedad, influyendo factores tales como: la capacidad del controlador para influir en la toma de decisiones, su disposición a monitorear el desempeño de la firma, el compromiso con la organización y sus empleados, su identidad corporativa y la responsabilidad social que asume. La posibilidad de identificar distintos intereses está motivada en el trabajo de Jiménez (2001), quien en su análisis de los grupos económicos chilenos se focaliza en las estrategias que siguen sus empresas, identificando que éstos presentan patrones recurrentes en la selección de industrias, recursos utilizados, modelos de negocios desarrollados y visión implícita, interviniendo esos cuatro factores en la definición de sus acciones y en su desempeño. Así, se plantean dos hipótesis que toman por referente principal a los controladores con un origen familiar. La primera, denominada hipótesis del compromiso familiar, señala que cuando el controlador corresponde a una familia con una historia empresarial, tal condición puede traducirse en un ambiente de mayor cooperación y diseminación de

1 Como evidencia al respecto, citamos tres casos emblemáticos en Chile que fueron motivo de análisis y estudios sobre los conflictos que pueden surgir entre controladores y accionistas minoritarios: (1) el Caso Chispas, donde el Grupo Yurascek traspasó a Endesa España el control de Enersis; (2) la transacción entre la española EBRO y el Grupo Pathfinder por el control de IANSA; y (3) el traspaso en CTC de una parte del negocio de Telefónica.Net a la filial de Telefónica España, operación que fue objetada por los accionistas minoritarios.

2 También resulta relevante considerar el quorum -de dos tercios de las acciones emitidas con derecho a voto- que se necesita para tomar decisiones en materias críticas para las sociedades anónimas, decisiones que un controlador que acumule al menos el $67 \%$ de la propiedad de la empresa podría adoptar en su propio beneficio. 
información al interior de la firma, junto con reflejar a clientes y proveedores un compromiso a largo plazo e identificación con el desempeño de los negocios de la empresa, factores que en su conjunto se van a traducir en una mayor valoración de la compañía. Esto se contrapone al control de un grupo de ejecutivos o de una multinacional que carezca de un vínculo que la relacione a la empresa, siendo ésta gencralmente un medio a valorizar en el corto y mediano plazo para luego desprenderse de su participación, acto que no necesariamente favorecerá a los accionistas minoritarios. ${ }^{3}$ La segunda hipótesis plantea que, cuando el controlador corresponde a una familia, éste transformará la compañía en una especie de feudo, ya que es probable que los objetivos de la empresa estén supeditados a sus valores personales, pudiendo primar valores familiares o factores de responsabilidad social, entre otros. A ello se puede sumar el mayor énfasis en proteger la privacidad (revelando poca información sobre las actividades internas de la empresa) y la falta de parámetros objetivos de selección de personal (lo que convertirá a la compañía en una fuente de empleo para familiares y amigos). Por último, es probable que se produzcan conflictos generacionales, problemas en la sucesión a los herederos, pérdida de dirección estratégica y confusión en los liderazgos, cuestiones que impactarán negativamente en la capacidad de la empresa para responder estratégicamente a los desafíos del entorno del negocio. Esto sería aún más probable cuando el accionista controlador es el fundador de la empresa.

El artículo se organiza en cuatro secciones: la sección dos explica la metodología utilizada; la sección tres presenta el análisis de los resultados y la última sección concluye el estudio.

\section{Datos y metodología}

La población inicial está compuesta por todas las sociedades anónimas abiertas vigentes a diciembre de 2002 para las cuales la Bolsa de Comercio de Santiago proporciona información, las que suman un total de 222 empresas. Para definir la muestra de estudio se aplicaron progresivamente los criterios excluyentes presentados en el Cuadro 1.

Para las 52 empresas de la muestra final se reunió la siguiente información anual relativa al período 1995-2002: Balance General, Estados de Resultados, Estado de Cambio en la Posición Financiera y Estado de Flujo de Efectivo; número de acciones en circulación y precio de cierre; número de accionistas comunes, filiales y coligadas; presencia bursátil. ${ }^{4}$ También se recopiló información con respecto al porcentaje de propiedad directa que mantenía el accionista (o pacto de accionistas) que ejercía como controlador de la empresa, y su identidad,

3 Como destacó en una reciente visita a Chile, Mariano Puig, Presidente del Holding PuigExea y del Grupo Europeo de Empresas Familiares, las empresas con base familiar tendrían mayor responsabilidad social y con el medio ambiente, mayor capacidad de tomar decisiones pensando en el largo plazo y desarrollan un sentido de pertenencia que al final se traduce en un mejor rendimiento que las acciones de multinacionales, quienes se caracterizan por un enfoque de corto plazo y actitudes en que sólo prima el beneficio económico

4 En caso de que la sociedad contara con más de una serie de acciones, se consideró la serie con mayor presencia bursátil. 
a fin de poder clasificarlo como familia, grupo de ejecutivos o multinacional. ${ }^{5}$

Cuadro 1. Criterios de selección de la muestra.

\begin{tabular}{|l|c|}
\hline \multicolumn{1}{|c|}{ Población de Sociedades Anónimas Abiertas } & 222 \\
\hline $\begin{array}{l}\text { Criterio 1: Presentar estados financieros en forma continua } \\
\text { para el período comprendido entre los años 1995 y 2002. }\end{array}$ & $(48)$ \\
\hline $\begin{array}{l}\text { Criterio 2: Corresponden a sociedades de inversión, sociedades financieras, } \\
\text { sociedades inmobiliarias y aquellas en proceso de liquidación, las que por } \\
\text { la naturaleza de su giro, activos y presentación de resultados no son } \\
\text { comparables a las empresas que tienen un giro productivo. }\end{array}$ & $(51)$ \\
\hline $\begin{array}{l}\text { Criterio 3: No registran movimiento bursátil en cada uno de los años } \\
\text { comprendidos en el período 1995 a 2002. }\end{array}$ & $(22)$ \\
\hline $\begin{array}{l}\text { Criterio 4. Sociedades que han diversificado significativamente en los rubros } \\
\text { financieros y de inversión, por las razones expuestas en el punto dos. }\end{array}$ & $(2)$ \\
\hline $\begin{array}{l}\text { Criterio 5: Sociedades con presencia bursátil inferior al 40\%, } \\
\text { para obtener una Q-Tobin lo más representativa posible. }\end{array}$ & $(47)$ \\
\hline \multicolumn{2}{|c|}{ Muestra de Sociedades Anónimas Abiertas Tratadas en el Estudio. } \\
\hline
\end{tabular}

Se escogió como medida del desempeño de la firma la Q-Tobin promedio, variable que también refleja las oportunidades de crecimiento del negocio. La $Q$-Tobin se define como el cociente entre el valor de mercado de la empresa y el costo de reposición de sus activos físicos. Para su cálculo se usó el siguiente proxy: ${ }^{6}$

$$
Q-\text { Tobin }=\frac{\text { Valor Mercado Patrimonio }+ \text { Valor Libro Deuda }}{\text { Valor Libro Activos }} .
$$

5 La principal fuente de información fue la nota de los estados financieros originada a partir de la Circular $N^{\circ} 792$ de 1988, donde se indica que cada empresa debe revelar el porcentaje de participación total y el número de accionistas que conforman su controlador. Para validar tal información, se cruzó con otras fuentes de datos, como la memoria de la empresa, la composición de su directorio y sus 12 mayores accionistas, además de hechos relevantes emitidas por la SVS, noticias publicadas en medios escritos de enfoque económico (Diario Financiero, Diario Estrategia, Revista Qué Pasa y Revista Capital, preferentemente) y diversos artículos ubicados en la red. El cruce de información permitió identificar la presencia de pactos entre accionistas no formalizados, y casos donde en la citada nota sólo se informaba el porcentaje de la sociedad con mayor participación de aquellas a través de las cuales el accionista mayoritario ejercía su control. En los casos donde existía un pacto controlador entre accionistas que se ubicaban en categorías distintas (familia, grupo de ejecutivos o multinacional), se tomó como origen el del integrante con mayor participación dentro del pacto o del que tenía a su cargo la administración, conservándose como porcentaje del controlador aquel mantenido por el total.

6 El valor de mercado del patrimonio para cada empresa se calculó multiplicando el número de acciones en circulación por el precio de cierre de la última transacción del año. En caso de existir más de una serie de acciones, se aplicó el mismo procedimiento a cada una de ellas, para luego sumar el total. Para los valores contables de la fórmula se usaron balances consolidados. En caso de no existir éstos, se utilizaron los balances individuales de la empresa. 
La $Q$-Tobin presenta ciertas ventajas con respecto a otras medidas de desempeño. Como destacan Lang \& Stulz (1994), al ser la Q-Tobin una medida de valoración que incorpora el valor presente de los flujos de caja futuros de la empresa, no es necesario hacer ajustes por nivel de riesgo o una normalización para poder comparar entre empresas, cualidad que no tienen otros indicadores como los retornos accionarios y las medidas contables, que también por su carácter histórico están sujetas al período de medición. Esta medida también ha sido aplicada por Morck et al. (1988). Sin embargo, se debe tener presente que la $Q$ Tobin se basa en supuestos sobre el mercado de capitales como son su eficiencia y agentes racionales con expectativas insesgadas. En ese contexto, la $Q$-Tobin reflejará el valor con que contribuyen los intangibles y las sinergias a la empresa, o la pérdida que significan los costos de agencia y el riesgo de expropiación. A ello se suma que el uso de un proxy va a estar sujeto a la discrecionalidad de los ajustes contables.

Se elaboraron tres medidas de diversificación: número de segmentos de negocios, el índice de Herfindahl y el índice de Entropía. Se tomó como parámetro de diversificación el nivel de ventas correspondiente a cada segmento, desagregándose su composición a partir de la clasificación $\mathrm{SIC},{ }^{7}$ al nivel de cuatro dígitos y, en los casos donde no se tuvo tal nivel de detalle, al nivel de dos dígitos. Los criterios de selección fueron: simplicidad, en el caso del primero; amplio uso, para el índice de Herfindahl; y mayor sensibilidad ante distintos niveles de diversificación, para el índice de Entropía (Jacquemim \& Berry, 1979). Además, las medidas de Herfindahl y Entropía destacan por sobre el número de segmentos, ya que en su formulación se considera ese antecedente y el grado en que se distribuyen las ventas entre los distintos negocios. El índice de Herfindahl se calcula según la expresión (2), donde $P_{i}$ es la participación del i-ésimo segmento en las ventas totales de la firma y $n$ corresponde al número de segmentos donde se diversifica la empresa.

$$
H=\sum_{i=1}^{n} P_{i} \times P_{i}, \quad \text { con } 0<H<1 .
$$

Esta expresión se reformuló tomándose su complemento, con el objeto de constituir un indicador creciente con el nivel de diversificación, vale decir:

$$
H^{C}=\left(1-\sum_{i=1}^{n} P_{i}^{2}\right), \quad \text { con } 0 \leq H^{C} \leq 1 .
$$

Por su parte, el índice de Entropía resulta directamente creciente con el grado de diversificación de la firma:

$$
\mathrm{E}=\sum_{i=1}^{n} P_{i} \times \ln \frac{1}{P_{i}}, \quad \text { con } 0 \leq \mathrm{E} \leq \ln (\mathrm{n}) .
$$

7 Se destaca que obtener las ventas por segmento en las empresas diversificadas demandó un intenso trabajo de campo, debiendo revisarse las memorias para cada una de las empresas de la muestra en la SVS. Donde en la mayoría de las ocasiones no constituyó un antecedente que se proporcionara en forma directa, sino que tuvo que derivarse a partir de los análisis a los estados financieros e información de subsidiarias. 
La variable grado de concentración de la propiedad (controlador) corresponde al porcentaje de propiedad directa que mantiene un accionista (o pacto de accionistas) a través de una o más sociedades, resultando su posesión en el ejercicio del control de la empresa. ${ }^{8}$

Para evitar conclusiones erradas, se incorporaron las siguientes variables de control: (a) LNACT: Logaritmo natural del valor contable de los activos. Es un proxy del tamaño de la empresa y de los activos intangibles que podrían estar correlacionados con esa medida (Lang \& Stulz, 1994; Morck et al., 1988); (b) RO/VTAS: cociente entre el resultado operacional y las ventas de la empresa. Es un proxy de la rentabilidad. Busca controlar la posibilidad de que el porcentaje del controlador sea mayor en empresas jóvenes conl altos márgenes, lo cual resultaría en distintos incentivos asociados a su administración (Morck et al., 1988); (c) $D / A C T$ : cociente entre el valor libro de la deuda a largo plazo y el valor de los activos de la firma. Podría capturar en parte el valor del ahorro tributario de la deuda; (d) DIV/ACT: cociente entre los dividendos pagados por la empresa en un año y el total de activos. ${ }^{9}$ Es un proxy para analizar si la empresa enfrenta racionamiento en los mercados financieros o no (Lang \& Stulz, 1994). Alternativamente, su magnitud podría indicar costos de agencia ya que, al distribuir fondos entre los accionistas, es menos probable que la empresa los destine a gastos pecuniarios o a inversiones ineficientes (Jensen, 1986); (e) GK/ACT: cociente entre los gastos de capital de la empresa y el total de activos. Es un proxy del grado en que la empresa está invirtiendo en oportunidades de crecimiento, lo cual permite evaluar -en complemento con RO/VTAS- la importancia de la hipótesis de eficiencia de mercados de capital interno (Hyland \& Diltz, 2002; Lins \& Servaes, 2002); (f) IND: Tasa de crecimiento anual del sector económico al cual pertenece la empresa. Busca controlar la posibilidad de que cambios en la Q-Tobin respondan a diferencias entre industrias en crecimiento y maduras, antes que a diferencias entre las empresas. Además, el modelo se extendió para controlar por condiciones macroeconómicas, incorporando tres variables dummy (D): una para cada uno de los años 1997, 1998 y 1999, período en que la crisis asiática tuvo su mayor impacto en Chile.

\section{A. Método estadístico de estimación}

En una primera etapa del análisis se utiliza un modelo econométrico asociado a datos de panel, del tipo balanceado. La principal ventaja de este modelo radica en la flexibilidad que entrega para analizar las diferencias de comportamiento

8 Se entiende por controlador la definición que se entrega en el artículo 97 de la Ley N 18.045 de Mercado de Valores, cuyo contenido se transcribe a continuación: "Es controlador de una sociedad toda persona o grupo de personas con acuerdo de actuación conjunta que, directamente o a través de otras personas naturales o jurídicas, participa en su propiedad y tiene poder para realizar alguna de las siguientes actuaciones:

a) Asegurar la mayoría de votos en las juntas de accionistas y elegir a la mayoría de los directores tratándose de sociedades anónimas, o asegurar la mayoría de votos en las asambleas o reuniones de sus miembros y designar al administrador o representante legal o a la mayoría de ellos, en otro tipo de sociedades, o

b)Influir decisivamente en la administración de la sociedad."

9 No se hizo distinción en relación a si tales dividendos fueron definitivos o eventuales. 
que pueden darse entre y dentro de los distintos grupos de empresas considerados, en vez de limitarse a un análisis transversal. El modelo queda formulado de la siguiente manera:

$$
\begin{aligned}
Q-\text { Tobin }_{i t} & =\mu_{i t}+\beta_{1} \text { Diversificacion }_{i t}+\beta_{2} \text { Control }_{i t}+\beta_{3} \mathrm{LNACT}_{i t} \\
& +\beta_{4} \mathrm{RO} / \mathrm{VTAS}_{i t}+\beta_{5} \mathrm{D} / \mathrm{ACT}_{i t}+\beta_{6} \mathrm{DIV} \mathrm{ACT}_{i t} \\
& +\beta_{7} \mathrm{GK} / \mathrm{ACT}_{i t}+\beta_{8} \mathrm{IND}_{i t}+D_{1997}+D_{1998}+D_{1999}+\varepsilon_{i t}
\end{aligned}
$$

con $i=1,2, \cdots, 52$ y $t=1995$ hasta 2002. En una segunda etapa se ejecuta el siguiente procedimiento, a fin de determinar la conveniencia de aplicar efectos fijos o aleatorios en la estimación: ${ }^{10}$ (1) contraste de significancia para los efectos de grupo bajo el modelo de efectos fijos, donde se analiza si los términos constante $\mu_{i}$ son similares o si existen diferencias estadísticamente significativas entre los grupos; (2) contraste sobre la presencia de efectos aleatorios a través del test multiplicador de Lagrange de Breusch \& Pagan; (3) contraste de especificación sobre la ortogonalidad de los efectos fijos y aleatorios a través del test de Hausman. La evidencia favoreció al modelo de efectos fijos. Esto se ajusta a la teoría, pues las empresas que componen la muestra no corresponden a una selección aleatoria. Además, como señalan Himmelberg et al. (1999), el uso de efectos fijos permite capturar heterogeneidad no observable de la firma que permanece constante en el tiempo, como son inversiones tecnológicas o calidad administrativa. A continuación se analiza la presencia de heterocedasticidad entre empresas, lo cual es bastante probable debido a que cada firma presenta características y riesgos distintos. El contraste del multiplicador de Lagrange evidencia heterocedasticidad de sección cruzada. Para dar cuenta de su presencia y evitar inferencias erradas, se estima el modelo con covarianza consistente de White para los errores.

En una cuarta etapa del análisis, se examina la relación entre las variables críticas del estudio: Q-Tobin, diversificación y grado de concentración de la propiedad. El objetivo es determinar si la vinculación de la Q-Tobin a esas dos variables responde a una especificación lineal, o si dicha relación debe modelarse incorporando posibles quiebres en su tendencia o efectos cuadráticos. Estas especificaciones pueden llevar a que las hipótesis bajo prueba encontraran validez empírica en tramos de medición, por lo que obviarlas, junto con llevar a conclusiones erróneas, podría significar perder valiosa información. ${ }^{11}$

En una quinta etapa se realiza un análisis por tramos. Siguiendo la metodología aplicada por Morck et al. (1988), cuyo objetivo principal es encontrar un modo parsimonioso de representar un patrón de datos no estrictamente lineal, se permiten dos cambios en los coeficientes de las variables diversificación y porcentaje del controlador. Como destacan los autores, seguir tal parametrización

10 Para una revisión teórica ver: Green ( $3^{\circ}$ Edición), capítulo 14; Novales ( $2^{\circ}$ Edición), capítulo 15.

11 Al respecto, Lins \& Servaes (2002) encontraron un fuerte apoyo a la hipótesis de expropiación cuando la administración concentra entre el $10 \%$ y el $30 \%$ de la propiedad, y Khanna \& Palepu (1999) reconocieron una relación cuadrática entre el desempeño de la empresa y el grado de diversificación del conglomerado al que pertenece. 
permite obtener resultados más confiables que usar variables dummy para estimar medias condicionales de la $Q$-Tobin en tramos de las variables relevantes.

En una sexta del análisis se examina el impacto de las transferencias de fondos entre empresas relacionadas. En esta fase se incorporan un conjunto de variables adicionales, con el propósito de verificar dos hipótesis que pueden asociarse a la presencia de transferencias financieras y comerciales entre empresas relacionadas: eficiencia de mercado de capitales interno y expropiación a minoritarios. Las nuevas variables son: (a) LNACCT: Logaritmo natural sobre el número de accionistas comunes presentes en la empresa a fines de cada año. Es un proxy de la dispersión de los accionistas minoritarios $\mathrm{y}$, con ello, de sus incentivos para monitorear a la administración; (b) CxC/AT: cociente entre los documentos y cuentas por cobrar (de corto y largo plazo) que se tiene con sociedades relacionadas y el total de activos. Es un proxy de la magnitud del mercado de capital interno en la empresa; (c) CxP/PS: Razón entre los documentos y cuentas por pagar (de corto y largo plazo) que se tiene con sociedades relacionadas y el total de pasivos exigibles. Es un proxy de la magnitud de las transacciones entre empresas relacionadas; (d) IER/ACT: Razón entre el monto invertido en empresas relacionadas y el activo total. Es un proxy del flujo de fondos entre subsidiarias y la matriz; (e) AFP: Variable dummy que toma el valor 1 si la empresa es aefepeable $e^{12}$ y 0 si no lo es, a fines de cada año de estudio. Es un proxy de calidad; (f) A 10: Variable dummy. Toma el valor 1 en los años 2001 y 2002 si la empresa optó por acogerse al artículo $10^{\circ}$ transitorio de la Ley $19.705,{ }^{13}$ en caso contrario toma el valor $0{ }^{14}(\mathrm{~g})$ PRES: Presencia bursátil, que representa el porcentaje de días hábiles en un año donde las transacciones totales diarias sobre la acción de la empresa han alcanzado un monto mínimo de 80 unidades de fomento. Busca controlar por un posible premio por liquidez en aquellas empresas con menor nivel de transacciones. Finalmente, se identificaron tres tipos de accionistas controladores, a fin de capturar los distintos incentivos y responsabilidades que los afectan: familiar, grupo de ejecutivos y multinacionales. Se tomó como referente a Majluf et al. (1998). Además, se incorpora la categoría multinacionales, dada su relevante presencia en el sector productivo chileno.

\section{Análisis de los resultados}

El Cuadro 2 presenta las variables del modelo, coll los estadísticos para las firmas focalizadas ${ }^{15}$ y diversificadas. Las empresas focalizadas representan casi

12 Sociedad anónima en que estaba autorizada la inversión de los recursos de los Fondos de Pensiones por la Comisión Clasificadora de Riesgo. Esta información se obtuvo desde las circulares emitidas sobre la materia por la Superintendencia de AFP.

13 Tal antecedente, obtenido desde la SVS, resultaba importante porque permite, a partir de 2001, distinguir entre aquellas sociedades donde el controlador tienen libertad para enajenar su participación de las que no.

14 Como sucede entre los años 1995 y 2000.

15 Para ello se definió como empresa focalizada aquella que concentra el $100 \%$ de sus ventas en un solo rubro, quedando como diversificada cuando sus ingresos provienen de dos o más negocios. El criterio de clasificación se basa en el hecho de que gran parte del efecto por diversificar se gesta cuando la empresa deja su core business, siendo marginal el impacto de diversificar a 2 o más segmentos (Lang \& Stulz (1994)). 
la mitad de la muestra total (45.7\%), y son significativamente más pequeñas que las firmas diversificadas en una relación de prácticamente 4:1 -en base a la mediana de sus activos-, lo cual se mantiene al controlar por el nivel de ventas. En cuanto a desempeño, las firmas focalizadas y las diversificadas se valoran por sobre el valor libro de sus activos -según el promedio y la mediana de la $Q$-Tobin-, siendo la valoración relativamente mayor en las empresas focalizadas. Lo anterior resulta consistente con el hecho de que éstas últimas presentan una mayor rentabilidad. También se observa que las firmas focalizadas presentan un menor nivel de endeudamiento, además de pagar significativamente menos dividendos. Por último, es importante notar la mayor concentración de propiedad en el controlador que distingue a las empresas focalizadas.

Cuadro 2: Resumen estadístico para cada submuestra de firmas: focalizadas y diversificadas.

\begin{tabular}{|c|c|c|c|c|c|c|}
\hline \multirow[t]{2}{*}{ Variable } & \multicolumn{2}{|c|}{ Firm. Focalizadas } & \multicolumn{2}{|c|}{ Firm. Diversificadas } & \multicolumn{2}{|c|}{ Diferencia $=$ (Div. - Foc. $)$} \\
\hline & Media & Mediana & Media & Mediana & Media* & Mediana $^{* *}$ \\
\hline Q-Tobin & 1.47 & 1.31 & 1.17 & 1.03 & $\begin{array}{l}-0.3 \\
(0.00)\end{array}$ & $\begin{array}{l}-0.28 \\
(0.00)\end{array}$ \\
\hline $\begin{array}{c}N^{O} \text { de seg- } \\
\text { mentos }\end{array}$ & 1 & 1 & 2.98 & 3.00 & $\begin{array}{c}1.98 \\
(0.00)\end{array}$ & $\begin{array}{c}2.00 \\
(0.00)\end{array}$ \\
\hline $\begin{array}{l}\text { Controla- } \\
\text { lador, }\end{array}$ & 0.69 & 0.67 & 0.55 & 0.56 & $\begin{array}{l}-0.14 \\
(0.00) \\
\end{array}$ & $\begin{array}{l}-0.11 \\
(0.00) \\
\end{array}$ \\
\hline $\begin{array}{l}\text { Total acti- } \\
\operatorname{vos}(\mathrm{MM} \$)\end{array}$ & 208.0 & 99.6 & 1080.0 & 380.0 & $\begin{array}{l}872.0 \\
(0.00) \\
\end{array}$ & $\begin{array}{l}280.4 \\
(0.00)\end{array}$ \\
\hline $\begin{array}{l}\text { Total ven- } \\
\text { tas (MM\$) }\end{array}$ & 100.0 & 48.8 & 364.0 & 198.0 & $\begin{array}{l}264.0 \\
(0.00)\end{array}$ & $\begin{array}{l}149.2 \\
(0.00)\end{array}$ \\
\hline $\begin{array}{c}\text { Leverage } \\
(\mathrm{LP} / \mathrm{ACT})\end{array}$ & 0.20 & 0.17 & 0.23 & 0.21 & $\begin{array}{c}0.03 \\
(0.09) \\
\end{array}$ & $\begin{array}{c}0.04 \\
(0.03)\end{array}$ \\
\hline $\begin{array}{c}\text { Rentabi- } \\
\text { lidad }\end{array}$ & 0.20 & 0.19 & 0.15 & 0.14 & $\begin{array}{l}-0.05 \\
(0.00) \\
\end{array}$ & $\begin{array}{l}-0.05 \\
(0.00) \\
\end{array}$ \\
\hline $\begin{array}{c}\text { Crecimien- } \\
\text { to }\end{array}$ & 0.066 & 0.054 & 0.069 & 0.050 & $\begin{array}{c}0.00 \\
(0.70) \\
\end{array}$ & $\begin{array}{c}0.00 \\
(0.90) \\
\end{array}$ \\
\hline $\begin{array}{c}\text { Pago de } \\
\text { dividendos }\end{array}$ & 0.066 & 0.030 & 0.027 & 0.020 & $\begin{array}{c}-0.039 \\
(0.00) \\
\end{array}$ & $\begin{array}{l}-0.01 \\
(0.00)\end{array}$ \\
\hline Total obs. & & & & 26 & & \\
\hline
\end{tabular}

${ }^{*} \mathrm{P}$-value del test $t$ para igualdad de medias en paréntesis. ${ }^{* *} \mathrm{P}$-value del test de Wilcoxon / Mann-Whitney para igualdad de medianas en paréntesis.

El Cuadro 3 presenta dos medidas de correlación. La primera es lineal y supone una distribución de probabilidades. La segunda es el ranking de correlación de Spearman, que es una medida no paramétrica y permite controlar por la presencia de una especificación distinta a la lineal. Se observa que ambas medidas resultan significativas al 1\%, salvo en la relación de la $Q$-Tobin con el porcentaje del controlador, donde el ranking de correlación de Spearman no resultó significativo al nivel del $10 \%$, pero sí la respectiva correlación lineal. Del cuadro 
se concluye que la diversificación es un elemento que afecta negativamente el desempeño de la empresa, y que esa diversificación es mayor cuando menos propiedad mantiene el controlador.

Cuadro 3: Medidas de correlación.

\begin{tabular}{|c|c|c|c|c|}
\hline \multicolumn{5}{|c|}{ Medidas de Correlación } \\
\hline & \multicolumn{2}{|c|}{$\mathrm{N}^{\circ}$ Segmentos } & \multicolumn{2}{|c|}{ Herfindalh } \\
\hline & $\begin{array}{c}\text { Lineal } \\
\text { (P-value) }\end{array}$ & $\begin{array}{l}\text { Spearman } \\
\text { (P-value) }\end{array}$ & $\begin{array}{c}\text { Lineal } \\
\text { (P-value) }\end{array}$ & $\begin{array}{l}\text { Spearman } \\
\text { (P-value) }\end{array}$ \\
\hline Q-Tobin & $\begin{array}{l}-0.17 \\
(0.00)\end{array}$ & $\begin{array}{l}-0.16 \\
(0.00)\end{array}$ & $\begin{array}{l}-0.18 \\
(0.00)\end{array}$ & $\begin{array}{l}-0.20 \\
(0.00)\end{array}$ \\
\hline$N^{\circ}$ Segmentos & 1.00 & 1.00 & $\begin{array}{c}0.87 \\
(0.00)\end{array}$ & $\begin{array}{c}0.93 \\
(0.00)\end{array}$ \\
\hline Herfindalh & & & 1.00 & 1.00 \\
\hline \multicolumn{5}{|l|}{ Entropía } \\
\hline Control & & & & \\
\hline \multicolumn{5}{|c|}{ Medidas de Correlación } \\
\hline & \multicolumn{2}{|c|}{ Entropía } & \multicolumn{2}{|c|}{ Control } \\
\hline & $\begin{array}{c}\text { Lineal } \\
\text { (P-value) }\end{array}$ & $\begin{array}{l}\text { Spearman } \\
\text { (P-value) }\end{array}$ & $\begin{array}{c}\text { Lineal } \\
\text { (P-value) }\end{array}$ & $\begin{array}{c}\text { Spearman } \\
\text { (P-value) }\end{array}$ \\
\hline Q-Tobin & $\begin{array}{l}-0.17 \\
(0.00)\end{array}$ & $\begin{array}{l}-0.18 \\
(0.00)\end{array}$ & $\begin{array}{c}0.19 \\
(0.00)\end{array}$ & $\begin{array}{c}0.07 \\
(0.13)\end{array}$ \\
\hline$N^{o}$ Segmentos & $\begin{array}{c}0.91 \\
(0.00) \\
\end{array}$ & $\begin{array}{c}0.95 \\
(0.00) \\
\end{array}$ & $\begin{array}{l}-0.44 \\
(0.00) \\
\end{array}$ & $\begin{array}{l}-0.43 \\
(0.00)\end{array}$ \\
\hline Herfindalh & 0.99 & 0.99 & $\begin{array}{l}-0.34 \\
(0.00)\end{array}$ & $\begin{array}{l}-0.38 \\
(0.00)\end{array}$ \\
\hline Entropía & 1.00 & 1.00 & $\begin{array}{l}-0.38 \\
(0.00)\end{array}$ & $\begin{array}{l}-0.39 \\
(0.00)\end{array}$ \\
\hline Control & & & $(1.00)$ & $(1.00)$ \\
\hline
\end{tabular}

Los resultados del modelo de panel (fórmula 5) son presentados en el Cuadro 4. Los coeficientes de las variables asociadas a la diversificación son positivos, aunque no significativos, lo que va en contra de la evidencia preliminar entregada por las correlaciones -donde la diversificación, en sus tres definiciones, resultó negativa y significativa-. También resulta contradictorio que el signo de la variable que controla por tamaño (logaritmo de los activos) resulte negativo y significativo, cuando la diversificación resultó positiva. Esto será estudiado cuando se analicen las variables por tramos.

El signo positivo y significativo del porcentaje del controlador concuerda con la evidencia provista por el análisis de correlaciones, proporcionando así apoyo a la hipótesis de convergencia de intereses, en desmedro de la hipótesis de expropiación. Los coeficientes de las variables rentabilidad y crecimiento 
resultaron, como era de esperarse, positivos y significativos. El coeficiente del leverage no es significativo, lo que denotaría que los beneficios que se logran por un mayor endeudamiento serían compensados por los costos asociados a un mayor apalancamiento financiero. También se observa que el desempeño de la empresa no se encuentra relacionado con el pago de dividendos. No obstante, al controlar por la industria a la que pertenece la empresa, ésta variable resulta altamente significativa.

Con respecto a las variables temporales, se debe señalar que el signo negativo y significativo asociado a los años 1997 y 1998 refleja el impacto que tuvo la crisis asiática en el mercado de valores chileno. Como estas variables no forman parte del estudio -más allá de controlar por shocks macroeconómicos y ciclos de negocios-, si bien se continuará controlando por ellas, se omitirá su presentación en las regresiones posteriores.

Cuadro 4: Resultados de la regresión: modelos I, II y III. Modelos de efectos fijos, con corrección de heterocedasticidad entre empresas y errores estándar por covarianzas consistentes de White $\mathrm{P}$-value consistente de White en paréntesis.

\begin{tabular}{|c|c|c|c|}
\hline Variables* & Modelo I & Modelo II & Modelo III \\
\hline$N^{\circ}$ Segmentos & $\begin{array}{l}0.2200 \\
(0.18) \\
\end{array}$ & & \\
\hline Herfindahl & & $\begin{array}{l}0.5143 \\
(0.13)\end{array}$ & \\
\hline Entropía & & & $\begin{array}{c}0.4613 \\
(0.12)\end{array}$ \\
\hline$\%$ Control & $\begin{array}{l}0.9393 \\
(0.01)\end{array}$ & $\begin{array}{l}0.8923 \\
(0.02)\end{array}$ & $\begin{array}{l}0.8990 \\
(0.01)\end{array}$ \\
\hline $\mathrm{LN}(\mathrm{ACT})$ & $\begin{array}{c}-0.5358 \\
(0.00)\end{array}$ & $\begin{array}{c}-0.5311 \\
(0.00)\end{array}$ & $\begin{array}{c}-0.5375 \\
(0.00)\end{array}$ \\
\hline RO/V'TAS & $\begin{array}{c}0.9566 \\
(0.02)\end{array}$ & $\begin{array}{l}0.9400 \\
(0.02)\end{array}$ & $\begin{array}{c}0.9738 \\
(0.02)\end{array}$ \\
\hline $\mathrm{GK} / \mathrm{ACT}$ & $\begin{array}{r}1.2441 \\
(0.00) \\
\end{array}$ & $\begin{array}{l}1.2091 \\
(0.00) \\
\end{array}$ & $\begin{array}{l}1.1844 \\
(0.00) \\
\end{array}$ \\
\hline $\mathrm{DIV} / \mathrm{ACT}$ & $\begin{array}{c}1.1048 \\
(0.32) \\
\end{array}$ & $\begin{array}{l}1.1347 \\
(0.31) \\
\end{array}$ & $\begin{array}{l}1.1243 \\
(0.31) \\
\end{array}$ \\
\hline B LP/ACT & $\begin{array}{c}-0.6071 \\
(0.15)\end{array}$ & $\begin{array}{c}-0.6024 \\
(0.16)\end{array}$ & $\begin{array}{c}-0.5846 \\
(0.17)\end{array}$ \\
\hline INDUSTRIA & $\begin{array}{l}1.9451 \\
(0.01) \\
\end{array}$ & $\begin{array}{l}1.8890 \\
(0.01) \\
\end{array}$ & $\begin{array}{l}1.8992 \\
(0.01) \\
\end{array}$ \\
\hline D1997 & $\begin{array}{c}-0.2182 \\
(0.00)\end{array}$ & $\begin{array}{c}-0.2163 \\
(0.00)\end{array}$ & $\begin{array}{c}-0.2167 \\
(0.00)\end{array}$ \\
\hline D1998 & $\begin{array}{c}-0.2658 \\
(0.00)\end{array}$ & $\begin{array}{c}-0.2569 \\
(0.00) \\
\end{array}$ & $\begin{array}{c}-0.2566 \\
(0.00) \\
\end{array}$ \\
\hline D1999 & $\begin{array}{l}0.0573 \\
(0.33) \\
\end{array}$ & $\begin{array}{r}0.0653 \\
(0.28) \\
\end{array}$ & $\begin{array}{l}0.0658 \\
(0.28) \\
\end{array}$ \\
\hline
\end{tabular}


Cuadro 4: (continuación).

\begin{tabular}{|c|c|c|c|}
\hline Variables* & Modelo I & Modelo II & Modelo III \\
\hline$R^{2}$ Ajustado & $61.1 \%$ & $60.7 \%$ & $60.9 \%$ \\
\hline F-statistic & 71.40 & 70.28 & 70.83 \\
\hline$P$ (F-statistic) & 0.00 & 0.00 & 0.00 \\
\hline
\end{tabular}

* Se omite la presentación de los efectos fijos.

En el análisis de causalidad por tramos, además de los cambios en los coeficientes de las variables diversificación y porcentaje del controlador, se incorporó el $\log (a c t)$ con el fin de encontrar evidencia que permita dar respuesta a la inconsistencia aparecida en la etapa anterior. Las variables presentadas en el Cuadro 5 representan los tramos que registraron la mejor bondad de ajuste.

Cuadro 5: Variables de diversificación, propiedad y tamaño, por tramo.

\begin{tabular}{|c|c|}
\hline \multicolumn{2}{|r|}{ VARIABLES DE DIVERSIFICACIÓN } \\
\hline \multicolumn{2}{|c|}{ Número de Segmentos } \\
\hline NUM-1 a 2 & $\begin{array}{l}=\text { Número de segmentos si estos son } \leq 2 \\
=2 \text { si número de segmentos }>2\end{array}$ \\
\hline NUM - 3 & $\begin{array}{l}=0 \text { si número de segmentos } \leq 2 \\
=\text { Número de segmentos menos } 2 \text { si } 2<\text { número de segmentos } \leq 3\end{array}$ \\
\hline NUM - +3 & $\begin{array}{l}=0 \text { si número de segmentos } \leq 3 \\
=\text { Número de segmentos menos } 3 \text { si número de segmentos }>3\end{array}$ \\
\hline \multicolumn{2}{|c|}{ Índice de Herfindahl: } \\
\hline $\mathrm{H}-0$ a 40 & $\begin{array}{l}=\text { Índice de Herfindahl si éste es } \leq 0.40 \\
=0.40 \mathrm{si} \text { índice de Herfindahl }>0.40\end{array}$ \\
\hline $\mathrm{H}-40$ a 60 & $\begin{array}{l}=0 \text { si índice de Herfindahl } \leq 0.40 \\
=\text { Índice de Herfindahl menos } 0.40 \text { si } 0.40<\text { índice de Herfindahl } \leq 0.60\end{array}$ \\
\hline $\mathrm{H}-+60$ & $\begin{array}{l}=0 \text { si índice de Herfindahl } \leq 0.60 \\
=\text { Índice de Herfindahì menos } 0.60 \text { si índice de Herfindahl }>0.60\end{array}$ \\
\hline \multicolumn{2}{|c|}{ Índice de Entropía: } \\
\hline $\mathrm{E}-0$ a 55 & $\begin{array}{l}=\text { Índice de Entropía si éste es } \leq 0.55 \\
=0.55 \text { si índice de Entropía }>0.55\end{array}$ \\
\hline E - 55 a 85 & $\begin{array}{l}=0 \text { si índice de Entropía } \leq 0.55 \\
=\text { Índice de Entropía menos } 0.55 \text { si } 0.55<\text { índice de Entropía } \leq 0.85\end{array}$ \\
\hline $\mathrm{E}-+85$ & $\begin{array}{l}=0 \text { si índice de Entropía } \leq 0.85 \\
=\text { Índice de Entropía menos } 0.85 \text { si índice de Entropía }>0.85\end{array}$ \\
\hline \multicolumn{2}{|r|}{ VARIABLES DE PROPIEDAD: Porcentaje del Controlador } \\
\hline$\%-0$ a 40 & $\begin{array}{l}=\% \text { Controlador si éste es } \leq 0.40 \\
=0.40 \text { si } \% \text { Controlador }>0.40\end{array}$ \\
\hline$\%-40$ a 65 & $\begin{array}{l}=0 \text { si } \% \text { Controlador } \leq 0.40 \\
=\% \text { Controlador menos } 0.40 \text { si } 0.40<\% \text { Controlador } \leq 0.65\end{array}$ \\
\hline$\%-+65$ & $\begin{array}{l}=0 \mathrm{si} \% \text { Controlador } \leq 0.65 \\
=\% \text { Controlador menos } 0.65 \text { si } \% \text { Controlador }>0.65\end{array}$ \\
\hline
\end{tabular}




\section{Cuadro 5 (continuación).}

\begin{tabular}{|cl|}
\hline$\%-0$ a 20 & VARIABLES DE TAMAÑO: $\log ($ activos $)$ \\
& $=\log ($ ACT $)$ si éste es $\leq 0.20$ \\
& $=0.20$ si $\log ($ ACT $)>0.20$ \\
& $=0$ si $\log (A C T) \leq 0.20$ \\
& $=\log ($ ACT $)$ menos 0.20 si $0.20<\log ($ ACT $) \leq 21.5$ \\
& $=0$ si $\log ($ ACT $) \leq 21.5$ \\
& $=\log ($ ACT $)$ menos 21.5 si $\log ($ ACT $)>21.5$
\end{tabular}

Los resultados del modelo anterior se presentan en el Cuadro 6. Para número de negocios y Entropía se observa un efecto positivo y significativo en el segmento intermedio, lo que señalaría que, para un determinado rango, la diversificación crea valor. Por el contrario, Herfindahl registra un efecto negativo y significativo en su tramo final, indicando que la diversificación destruye valor a partir de cierto nivel. En consecuencia, los resultados no son concluyentes con respecto a la diversificación y su efecto sobre el valor de la firma.

En relación con el porcentaje del controlador, se observa que en su tramo inicial el coeficiente es negativo aunque no significativo, lo que podría indicar que los beneficios por una alineación de intereses son compensados por el consumo no pecuniario del controlador para bajos niveles de propiedad. No obstante, cuando la participación del controlador es mayor al $40 \%$, se observa un efecto positivo y significativo para número de negocios y Herfindahl (a un nivel de significancia del 5\%), y para Entropía (al 10\%), concordando con la hipótesis de alineación de intereses. Para niveles de concentración superiores al $65 \%$, existe una débil evidencia de que continúa manteniéndose un efecto positivo sobre el desempeño de la empresa cuando el controlador incrementa su participación. El menor efecto podría atribuirse a que comienza a ser importante el riesgo de expropiación al que quedan expuestos los minoritarios.

Cuadro 6: P-value consistente de White en paréntesis.

\begin{tabular}{|c|c|c|c|}
\hline Variables* & Modelo I & Modelo II & Modelo III \\
\hline NUM-1 a 2 & -0.0743 & & \\
& $(0.57)$ & & \\
NUM-3 & 0.7281 & & \\
& $(0.05)$ & & \\
NUM-+3 & -0.0412 & & \\
& $(0.70)$ & & \\
H-0 a 40 & & 0.0838 & \\
& & $(0.82)$ & \\
H-40 a 60 & & 3.0465 & \\
& & $(0.11)$ & \\
H-+60 & & -2.4023 & \\
& & $(0.05)$ & \\
E-0 a 55 & & & -0.2235 \\
& & & $(0.49)$ \\
\hline
\end{tabular}


6. (continuación).

\begin{tabular}{|c|c|c|c|}
\hline Variables* & Modelo I & Modelo II & Modelo III \\
\hline \multirow[t]{2}{*}{ E-55 a 85} & & & 2.4658 \\
\hline & & & $(0.04)$ \\
\hline \multirow{2}{*}{$\mathrm{E}-+85$} & & & -0.2470 \\
\hline & & & $(0.54)$ \\
\hline \multirow[t]{2}{*}{$\%-0$ a 40} & -1.6984 & -1.5623 & -1.1849 \\
\hline & $(0.21)$ & $(0.23)$ & $(0.37)$ \\
\hline \multirow{2}{*}{$\%-40$ a 65} & 1.4700 & 1.3382 & 1.0892 \\
\hline & $(0.03)$ & $(0.03)$ & $(0.09)$ \\
\hline \multirow[t]{2}{*}{$\%-+65$} & 0.7766 & 0.9526 & 1.0209 \\
\hline & $(0.16)$ & $(0.10)$ & $(0.09)$ \\
\hline \multirow[t]{2}{*}{ A- 0 a 20} & -0.7809 & -0.7645 & -0.7795 \\
\hline & $(0.00)$ & $(0.00)$ & $(0.00)$ \\
\hline \multirow[t]{2}{*}{$\mathrm{A}-20$ a 21.5} & 0.0378 & 0.0598 & 0.1324 \\
\hline & $(0.79)$ & $(0.68)$ & $(0.39)$ \\
\hline \multirow[t]{2}{*}{$\mathrm{A}-+21.5$} & -0.0267 & -0.2732 & -0.2833 \\
\hline & $(0.88)$ & $(0.02)$ & $(0.01)$ \\
\hline \multirow[t]{2}{*}{ RO/VTAS } & 0.8946 & 1.0229 & 0.9908 \\
\hline & $(0.03)$ & $(0.03)$ & $(0.03)$ \\
\hline \multirow[t]{2}{*}{$\mathrm{GK} / \mathrm{ACT}$} & 1.1127 & 1.3042 & 1.1761 \\
\hline & $(0.00)$ & $(0.00)$ & $(0.00)$ \\
\hline \multirow[t]{2}{*}{$\mathrm{DIV} / \mathrm{ACT}$} & 0.8272 & 1.0132 & 0.9197 \\
\hline & $(0.39)$ & $(0.35)$ & $(0.37)$ \\
\hline \multirow[t]{2}{*}{ B LP/ACT } & -0.7107 & -0.7005 & -0.7785 \\
\hline & $(0.10)$ & $(0.11)$ & $(0.07)$ \\
\hline \multirow[t]{2}{*}{ Industria } & 1.9170 & 1.8181 & 1.8905 \\
\hline & $(0.00)$ & $(0.01)$ & $(0.00)$ \\
\hline$R^{2}$ Ajustado & $63.9 \%$ & $62.5 \%$ & $63.2 \%$ \\
\hline F-statistic & 50.07 & 47.43 & 48.77 \\
\hline $\mathrm{P}$ (F-statistic) & 0.00 & 0.00 & 0.00 \\
\hline
\end{tabular}

* Se omite la presentación de los efectos fijos y temporales.

Para los tramos relativos al tamaño, la evidencia general apunta a una relación negativa y significativa entre tal variable y el desempeño de la empresa, con sólo un tramo menor donde no se presentarían efectos significativos. Para las variables restantes se continúa manteniendo el signo y significancia, salvo en el caso de la razón deuda largo plazo sobre activos totales, la cual resulta ser negativa y débilmente significativa.

También se analiza el impacto de las transferencias de fondos entre empresas relacionadas. El Cuadro 7 contiene en su parte superior la información del Cuadro 2, para fines comparativos, mientras que en su parte inferior se ubica aquello de interés para esta etapa. Se destaca que las empresas diversificadas 
tienden a otorgar mayores montos en créditos a sus relacionadas en comparación con las focalizadas. Por otra parte, el nivel de créditos que solicitan las firmas focalizadas se eleva hasta igualar el nivel de las empresas diversificadas.

Cuadro 7: Resumen estadístico.

\begin{tabular}{|c|c|c|c|c|c|c|}
\hline \multirow[t]{2}{*}{ Variable } & \multicolumn{2}{|c|}{ Firm. Focalizadas } & \multicolumn{2}{|c|}{ Firm. Diversificadas } & \multicolumn{2}{|c|}{ Diferencia =(Div. - Foc. $)$} \\
\hline & Media & Mediana & Media & Mediana & Media* & Mediana** $^{* *}$ \\
\hline Q-Tobin & 1.47 & 1.31 & 1.17 & 1.03 & $\begin{array}{l}-0.3 \\
(0.00)\end{array}$ & $\begin{array}{l}-0.28 \\
(0.00)\end{array}$ \\
\hline $\begin{array}{l}N^{a} \text { seg- } \\
\text { mentos }\end{array}$ & 1 & 1 & 2.98 & 3.00 & $\begin{array}{c}1.98 \\
(0.00)\end{array}$ & $\begin{array}{c}2.00 \\
(0.00)\end{array}$ \\
\hline $\begin{array}{l}\text { Contro- } \\
\text { lador }\end{array}$ & 0.69 & 0.67 & 0.55 & 0.56 & $\begin{array}{l}-0.14 \\
(0.00)\end{array}$ & $\begin{array}{l}-0.11 \\
(0.00)\end{array}$ \\
\hline $\begin{array}{l}\text { Total acti- } \\
\operatorname{vos}(\mathrm{MM} \$)\end{array}$ & 208.0 & 99.6 & 1080.0 & 380.0 & $\begin{array}{l}872.0 \\
(0.00)\end{array}$ & $\begin{array}{c}280.4 \\
(0.00)\end{array}$ \\
\hline $\begin{array}{l}\text { Total ven- } \\
\operatorname{tas}(\mathrm{MM} \$)\end{array}$ & 100.0 & 48.8 & 364.0 & 198.0 & $\begin{array}{l}264.0 \\
(0.00)\end{array}$ & $\begin{array}{l}149.2 \\
(0.00)\end{array}$ \\
\hline $\begin{array}{c}\text { Leverage } \\
(\mathrm{LP} / \mathrm{ACT})\end{array}$ & 0.20 & 0.17 & 0.23 & 0.21 & $\begin{array}{c}0.03 \\
(0.09)\end{array}$ & $\begin{array}{c}0.04 \\
(0.03)\end{array}$ \\
\hline $\begin{array}{l}\text { Rentabi- } \\
\text { lidad }\end{array}$ & 0.20 & 0.19 & 0.15 & 0.14 & $\begin{array}{l}-0.05 \\
(0.00)\end{array}$ & $\begin{array}{l}-0.05 \\
(0.00)\end{array}$ \\
\hline $\begin{array}{c}\text { Crecimien- } \\
\text { to }\end{array}$ & 0.066 & 0.054 & 0.069 & 0.050 & $\begin{array}{c}0.00 \\
(0.70)\end{array}$ & $\begin{array}{c}0.00 \\
(0.90)\end{array}$ \\
\hline $\begin{array}{c}\text { Pago de } \\
\text { dividendos }\end{array}$ & 0.066 & 0.030 & 0.027 & 0.020 & $\begin{array}{c}-0.039 \\
(0.00)\end{array}$ & $\begin{array}{l}-0.01 \\
(0.00)\end{array}$ \\
\hline $\begin{array}{c}\mathrm{C} \times \mathrm{C} \\
\text { Real/Act. }\end{array}$ & 0.052 & 0.016 & 0.118 & 0.077 & $\begin{array}{l}0.066 \\
(0.00)\end{array}$ & $\begin{array}{l}0.061 \\
(0.00)\end{array}$ \\
\hline $\begin{array}{l}\text { C x P } \\
\text { Real/Pas. }\end{array}$ & 0.163 & 0.054 & 0.186 & 0.078 & $\begin{array}{l}0.023 \\
(0.32)\end{array}$ & $\begin{array}{l}0.024 \\
(0.02)\end{array}$ \\
\hline IER/Act. & 0.120 & 0.047 & 0.460 & 0.454 & $\begin{array}{l}0.340 \\
(0.00)\end{array}$ & $\begin{array}{l}0.417 \\
(0.00)\end{array}$ \\
\hline Aefepeables & 0.72 & 1.00 & 0.96 & 1.00 & $\begin{array}{c}0.24 \\
(0.00)\end{array}$ & $\begin{array}{c}0.00 \\
(0.00)\end{array}$ \\
\hline $\begin{array}{l}\text { Acogidas } \\
\text { Art. } 10\end{array}$ & 0.13 & 0.00 & 0.12 & 0.00 & $\begin{array}{l}-0.01 \\
(0.94) \\
\end{array}$ & $\begin{array}{c}0.00 \\
(0.97) \\
\end{array}$ \\
\hline Presencia & 0.67 & 0.69 & 0.84 & 0.92 & $\begin{array}{c}0.17 \\
(0.00)\end{array}$ & $\begin{array}{c}0.23 \\
(0.00)\end{array}$ \\
\hline $\begin{array}{c}N^{a} \text { Accio- } \\
\text { nistas }\end{array}$ & 2,370 & 1,378 & 7,255 & 3,475 & $\begin{array}{l}4,885 \\
(0.00) \\
\end{array}$ & $\begin{array}{l}2,097 \\
(0.00)\end{array}$ \\
\hline Total obs. & & 0 & & 26 & & \\
\hline
\end{tabular}

${ }^{*} \mathrm{P}$-value del test $t$ para igualdad de medias en paréntesis. ${ }^{* *} \mathrm{P}$-value del test de Wilcoxon / Mann-Whitney para igualdad de medianas en paréntesis.

Se aprecia que existe una mayor liquidez en las acciones de las empresas diversificadas, lo que está en línea con la mayor dispersión de la propiedad y el 
menor porcentaje mantenido por el accionista controlador. Además, no se observa una clara diferencia entre empresas focalizadas y diversificadas en lo que dice relación con ser aefepeables o estar acogidas al artículo $10^{\circ}$ transitorio de la Ley 19.705.

El Cuadro 8 presenta los resultados de la regresión de panel en que se controla por tramos y se incorporan los nuevos elementos de interés. En general, no se ven alterados los signos de los coeficientes ni su significancia (en relación a los presentados en el Cuadro 6), salvo en el tramo superior del porcentaje del controlador (más de 65\%), el que resulta significativo al 1\%. Ello podría deberse a que se captura el riesgo de expropiación con una variable dummy. Para examinar tal posibilidad se incorpora en la regresión anterior una interacción entre lasvariables propiedad del controlador por sobre $65 \%$ y la dummy del artículo $10^{\circ},{ }^{16}$ encontrándose un coeficiente negativo y significativo -con valores (y p-values) de $-1.53(0.01),-1.87(0.01)$ y $-1.74(0.01)$, para las regresiones con número de segmentos, Herfindal y Entropía, respectivamente-, lo que señala que la concentración de la propiedad tiene un efecto negativo cuando está asociada al riesgo de expropiación. También se observa que aquellas variables asociadas con movimientos de flujo entre la empresa y sus relacionadas tienen un efecto negativo sobre la $Q$-Tobin, aunque sólo resultan ser significativos los créditos que se obtienen desde contrapartes con las cuales se tiene alguna vinculación. En cuanto a la dispersión de la propiedad, tiene el signo esperado, pero no es significativa. En conjunto, existe una débil evidencia a favor de que los flujos internos de la empresa constituyen un instrumento que permite expropiar riqueza a los minoritarios, antes que reflejar un mercado de capital interno eficiente. Finalmente, las demás variables muestran el signo esperado (aefepeable, artículo $10^{\circ}$ y presencia), pero sólo la presencia resulta significativa.

Cuadro 8: P-value consistente de White en paréntesis.

\begin{tabular}{|c|c|c|c|}
\hline Variables* & Modelo I & Modelo II & Modelo III \\
\hline NUM-1 a 2 & $\begin{array}{c}-0.0317 \\
(0.82)\end{array}$ & & \\
\hline NUM-3 & $\begin{array}{c}0.7992 \\
(0.03)\end{array}$ & & \\
\hline NUM-+3 & $\begin{array}{c}-0.0716 \\
(0.39)\end{array}$ & & \\
\hline H-0 a 40 & & 0.2360 & \\
& & $(0.50)$ & \\
\hline H-40 a 60 & & 2.6502 & \\
& & $(0.17)$ & \\
\hline H-+60 & & -2.5503 & \\
\hline E-0 a 55 & & $(0.05)$ & \\
\hline E-55 a 85 & & & -0.0533 \\
& & & $(0.88)$ \\
\hline
\end{tabular}

16 Regresiones no presentadas. Los resultados se pueden solicitar al autor. 
Cuadro 8 (continuación).

\begin{tabular}{|c|c|c|c|}
\hline Variables* & Modelo I & Modelo II & Modelo III \\
\hline $\mathrm{E}-+85$ & & & $\begin{array}{c}-0.3446 \\
(0.39)\end{array}$ \\
\hline$\%-0$ a 40 & $\begin{array}{c}-2.0781 \\
(0.13) \\
\end{array}$ & $\begin{array}{c}-1.8923 \\
(0.16) \\
\end{array}$ & $\begin{array}{c}-1.5668 \\
(0.26)\end{array}$ \\
\hline$\%-40$ a 65 & $\begin{array}{c}.5362 \\
(0.02) \\
\end{array}$ & $\begin{array}{c}1.3473 \\
(0.03) \\
\end{array}$ & $\begin{array}{l}1.1097 \\
(0.09) \\
\end{array}$ \\
\hline$\%-+65$ & $\begin{array}{l}1.2510 \\
(0.01) \\
\end{array}$ & $\begin{array}{l}1.4611 \\
(0.01) \\
\end{array}$ & $\begin{array}{l}1.5005 \\
(0.01) \\
\end{array}$ \\
\hline RO/VTAS & $\begin{array}{l}0.5412 \\
(0.16) \\
\end{array}$ & $\begin{array}{c}0.6774 \\
(0.14) \\
\end{array}$ & $\begin{array}{l}0.6641 \\
(0.13) \\
\end{array}$ \\
\hline $\mathrm{GK} / \mathrm{ACT}$ & $\begin{array}{l}0.9497 \\
(0.01) \\
\end{array}$ & $\begin{array}{l}1.1919 \\
(0.00) \\
\end{array}$ & $\begin{array}{c}1.0658 \\
(0.00) \\
\end{array}$ \\
\hline$\overline{\mathrm{DIV} / \mathrm{ACT}}$ & $\begin{array}{c}0.7013 \\
(0.37) \\
\end{array}$ & $\begin{array}{l}0.9485 \\
(0.31) \\
\end{array}$ & $\begin{array}{c}0.8686 \\
(0.33) \\
\end{array}$ \\
\hline B LP/ACT & $\begin{array}{c}-0.5274 \\
(0.19) \\
\end{array}$ & $\begin{array}{c}-0.5197 \\
(0.21)\end{array}$ & $\begin{array}{c}-0.6045 \\
(0.14) \\
\end{array}$ \\
\hline Industria & $\begin{array}{l}2.1615 \\
(0.00) \\
\end{array}$ & $\begin{array}{l}2.0310 \\
(0.01) \\
\end{array}$ & $\begin{array}{l}2.0819 \\
(0.00) \\
\end{array}$ \\
\hline $\mathrm{A}-0$ a 20 & $\begin{array}{c}-0.6148 \\
(0.00)\end{array}$ & $\begin{array}{c}-0.6263 \\
(0.00)\end{array}$ & $\begin{array}{c}-0.6437 \\
(0.00)\end{array}$ \\
\hline $\mathrm{A}-20$ a 21.5 & $\begin{array}{l}0.0275 \\
(0.83) \\
\end{array}$ & $\begin{array}{l}0.0563 \\
(0.68) \\
\end{array}$ & $\begin{array}{r}0.1318 \\
(0.68) \\
\end{array}$ \\
\hline $\mathrm{A}-+21.5$ & $\begin{array}{c}-0.0224 \\
(0.91) \\
\end{array}$ & $\begin{array}{c}-0.2846 \\
(0.03)\end{array}$ & $\begin{array}{c}-0.2798 \\
(0.03) \\
\end{array}$ \\
\hline CxC Rel. & $\begin{array}{c}-0.1031 \\
(0.73) \\
\end{array}$ & $\begin{array}{l}0.0817 \\
(0.80) \\
\end{array}$ & $\begin{array}{l}0.1180 \\
(0.71) \\
\end{array}$ \\
\hline CxP Rel. & -0.2627 & -0.2709 & -0.2545 \\
\hline IER/ACT & $\begin{array}{c}-0.1669 \\
(0.34)\end{array}$ & $\begin{array}{c}-0.1565 \\
(0.38)\end{array}$ & $\begin{array}{c}-0.1983 \\
(0.26)\end{array}$ \\
\hline $\operatorname{Ln}(\mathrm{ACCT})$ & $\begin{array}{c}-0.2032 \\
(0.19)\end{array}$ & $\begin{array}{c}-0.1742 \\
(0.29)\end{array}$ & $\begin{array}{c}-0.1784 \\
(0.28)\end{array}$ \\
\hline PRES & $\begin{array}{r}0.9567 \\
(0.00) \\
\end{array}$ & $\begin{array}{c}0.8101 \\
(0.00) \\
\end{array}$ & $\begin{array}{l}0.7606 \\
(0.00) \\
\end{array}$ \\
\hline Aefepeable & $\begin{array}{l}0.0460 \\
(0.63) \\
\end{array}$ & $\begin{array}{l}0.0829 \\
(0.39) \\
\end{array}$ & $\begin{array}{l}0.0921 \\
(0.35) \\
\end{array}$ \\
\hline Art.10 & $\begin{array}{c}-0.0592 \\
(0.38)\end{array}$ & $\begin{array}{c}-0.0440 \\
(0.54)\end{array}$ & $\begin{array}{c}-0.0631 \\
(0.40)\end{array}$ \\
\hline$R^{2}$ Ajustado & $66.2 \%$ & $64.2 \%$ & $64.9 \%$ \\
\hline F-statistic & 38.58 & 35.66 & 36.57 \\
\hline $\mathrm{P}$ (F-statistic) & 0.00 & 0.00 & 0.00 \\
\hline
\end{tabular}

* Se omite la presentación de los efectos fijos y temporales. Modelos I:

$N^{\circ}$ de segmentos; II: Herfindahl; III: Entropía. 
El Cuadro 9 presenta un análisis segmentado de la muestra, atendiendo al tipo de controlador. Prácticamente no existe distinción entre las distintas administraciones basándose en el desempeño, característica que se repite en el caso del crecimiento y del artículo $10^{\circ}$ transitorio. Por otro lado, sí existe una clara separación en la concentración de propiedad de las empresas, la cual decrece desde las multinacionales, seguido por las empresas familiares, para terminar con las controladas por grupo de ejecutivos.

Cuadro 9: Análisis segmentado por tipo de controlador.

\begin{tabular}{|c|c|c|c|c|c|c|}
\hline & \multicolumn{6}{|c|}{ Tipos de dueños de empresas } \\
\hline & \multicolumn{2}{|c|}{ Familias } & \multicolumn{2}{c|}{ Ejecutivos } & \multicolumn{2}{c|}{ Multinacionales } \\
\hline & Media & Mediana & Media & Mediana & Media & Mediana \\
\hline Q-Tobin & 1.25 & 1.05 & 1.36 & $1.20^{*}$ & 1.32 & 1.15 \\
\hline No. segmentos & 2.16 & 2.00 & $2.25^{\circ}$ & $2.00^{\circ}$ & $1.74^{*}$ & $1.00^{*}$ \\
\hline Controlador & 0.63 & 0.61 & $0.53^{* \circ}$ & $0.56^{* \circ}$ & $0.69^{*}$ & $0.70^{*}$ \\
\hline No. accionistas & 4,325 & 2,438 & $4,861^{\circ}$ & $2,381^{\circ}$ & $6,302^{*}$ & $2,786^{*}$ \\
\hline $\begin{array}{c}\text { Total activos } \\
\text { (MM } \$ \text { ) }\end{array}$ & 446.00 & 170.00 & $480.00^{\circ}$ & $209.00^{\circ}$ & $1280.00^{*}$ & $350.00^{*}$ \\
\hline $\begin{array}{c}\text { Total ventas } \\
\text { (MM } \$ \text { ) }\end{array}$ & 233.00 & 74.30 & $190.00^{\circ}$ & 98.90 & $323.00^{*}$ & $106.00^{*}$ \\
\hline $\begin{array}{c}\text { Leverage } \\
\text { (LP/ACT) }\end{array}$ & 0.16 & 0.15 & $0.23^{* \circ}$ & $0.21^{* \circ}$ & $0.28^{*}$ & $0.32^{*}$ \\
\hline Rentabilidad & 0.145 & 0.143 & $0.182^{*}$ & $0.163^{*}$ & $0.195^{*}$ & $0.191^{*}$ \\
\hline Crecimiento & 0.069 & 0.053 & 0.078 & 0.054 & 0.063 & 0.051 \\
\hline CxCReal/Act. & 0.063 & 0.036 & $0.102^{*}$ & $0.038^{*}$ & $0.108^{*}$ & $0.079^{*}$ \\
\hline CxPReal/Pas. & 0.192 & 0.072 & $0.121^{* \circ}$ & $0.047^{* \circ}$ & 0.214 & 0.105 \\
\hline IER/Activos & 0.42 & 0.041 & $0.23^{*}$ & $0.16^{*}$ & $0.22^{*}$ & $0.12^{*}$ \\
\hline Aefepeables & 0.80 & 1.00 & $0.95^{* \circ}$ & $1.00^{* \circ}$ & 0.81 & 1.00 \\
\hline Acogidas art.10 & 0.14 & 0.00 & $0.06^{*}$ & 0.00 & 0.18 & 0.00 \\
\hline Presencia & 0.74 & 0.80 & $0.81^{* \circ}$ & $0.90^{* \circ}$ & 0.75 & 0.81 \\
\hline Total obs. & & 172 & & 133 & & 111 \\
\hline
\end{tabular}

* Significativos al nivel de 10\% o menos de Ejecutivos/Multinacional r/a Familias.

- Significativos al nivel de $10 \%$ o menos de Ejecutivos r/a Multinacional.

Test de Medias: Test $t$; Test de Medianas: Wilcoxon / Mann-Whitney.

Del cuadro se desprende que las multinacionales controlan las empresas más grandes medidas por activos y ventas, presentan el mayor nivel de leverage, y tienen mayor dispersión de propiedad que las empresas controladas por ejecutivos o familias. Por su parte, las empresas controladas por familias presentan una menor rentabilidad, y un alto porcentaje de sus activos está conformado por inversiones en empresas relacionadas. Finalmente, las empresas dirigidas por ejecutivos se distinguen por ser aefepeables en un alto porcentaje, lo que explicaría el hecho de ser también las que ostentan la mayor presencia bursátil al contar con la demanda de los inversionistas institucionales. 
Para el análisis econométrico se adoptaron dos estrategias. Primero se incorporó una variable dummy que distinga a cada tipo de administración. Segundo, se conformaron paneles de datos para cada tipo de propiedad (desbalanceados), a fin de examinar el comportamiento que tienen las variables diversificación y propiedad en cada grupo particular. En ambos casos se tomó como referente el modelo por tramos expuesto en el Cuadro 6. El Cuadro 10 presenta los resultados de la primera estrategia. Familias y ejecutivos tienen un efecto positivo y significativo al compararse con las multinacionales, siendo el controlador de tipo familiar el que posee un mayor coeficiente. Con respecto a las demás variables, sus signos y niveles de significancia no presentan mayores cambios. Tampoco es posible obtener alguna conclusión respecto del efecto que tiene la diversificación sobre el desempeño de la empresa.

Cuadro 10: P-value consistente de White en paréntesis.

\begin{tabular}{|c|c|c|c|}
\hline Variables* & Modelo I & Modelo II & Modelo III \\
\hline \multirow[t]{2}{*}{ Familia } & 0.5661 & 0.5449 & 0.5549 \\
\hline & $(0.03)$ & $(0.04)$ & $(0.04)$ \\
\hline \multirow[t]{2}{*}{ Ejecutivos } & 0.2525 & 0.2760 & 0.2808 \\
\hline & $(0.00)$ & $(0.00)$ & $(0.00)$ \\
\hline \multirow[t]{2}{*}{ NUM-1 a 2} & -0.0815 & & \\
\hline & $(0.52)$ & & \\
\hline \multirow[t]{2}{*}{ NUM-3 } & 0.7078 & & \\
\hline & $(0.07)$ & & \\
\hline \multirow[t]{2}{*}{ NUM-+3 } & -0.0550 & & \\
\hline & $(0.58)$ & & \\
\hline \multirow[t]{2}{*}{$\mathrm{H}-0$ a 40} & & 0.0667 & \\
\hline & & $(0.85)$ & \\
\hline \multirow[t]{2}{*}{$\mathrm{H}-40$ a 60} & & 2.9284 & \\
\hline & & $(0.13)$ & \\
\hline \multirow[t]{2}{*}{$\mathrm{H}-+60$} & & -1.9342 & \\
\hline & & $(0.13)$ & \\
\hline \multirow[t]{2}{*}{$\mathrm{E}-0$ a 55} & & & -0.2589 \\
\hline & & & $(0.41)$ \\
\hline \multirow[t]{2}{*}{ E-55 a 85} & & & 2.4555 \\
\hline & & & $(0.04)$ \\
\hline \multirow[t]{2}{*}{$\mathrm{E}-+85$} & & & -0.2317 \\
\hline & & & $(0.58)$ \\
\hline \multirow[t]{2}{*}{$\%-0$ a 40} & -0.5932 & -0.4099 & -0.0171 \\
\hline & $(0.62)$ & $(0.72)$ & $(0.99)$ \\
\hline \multirow[t]{2}{*}{$\%-40$ a 65} & 1.2465 & 1.1357 & 0.8911 \\
\hline & $(0.04)$ & $(0.05)$ & $(0.13)$ \\
\hline \multirow[t]{2}{*}{$\%-+65$} & 0.4447 & 0.6593 & 0.7194 \\
\hline & $(0.37)$ & $(0.24)$ & $(0.21)$ \\
\hline \multirow[t]{2}{*}{$A-0$ a 20} & -0.6968 & -0.6809 & -0.6947 \\
\hline & $(0.00)$ & $(0.00)$ & $(0.00)$ \\
\hline
\end{tabular}


Cuadro 10 (continuación).

\begin{tabular}{|c|c|c|c|}
\hline Variables* & Modelo I & Modelo II & Modelo III \\
\hline A-20 a 21.5 & 0.0956 & 0.1150 & 0.1941 \\
& $(0.47)$ & $(0.40)$ & $(0.19)$ \\
A-+21.5 & 0.0650 & -0.1653 & -0.1766 \\
& $(0.71)$ & $(0.17)$ & $(0.13)$ \\
RO/VTAS & 0.8971 & 1.0211 & 0.9940 \\
& $(0.03)$ & $(0.03)$ & $(0.03)$ \\
GK/ACT & 1.1075 & 1.3024 & 1.1751 \\
& $(0.00)$ & $(0.00)$ & $(0.00)$ \\
DIV/ACT & 0.7704 & 1.9320 & 0.8381 \\
& $(0.40)$ & $(0.36)$ & $(0.39)$ \\
B LP/ACT & -0.5522 & -0.5438 & -0.6202 \\
& $(0.15)$ & $(0.16)$ & $(0.11)$ \\
Industria & 2.0863 & 2.0101 & 2.0845 \\
& $(0.00)$ & $(0.00)$ & $(0.00)$ \\
\hline$R^{2}$ Ajustado & $65.1 \%$ & $63.7 \%$ & $64.5 \%$ \\
F-statistic & 46.81 & 44.36 & 45.75 \\
P(F-statistic) & 0.00 & 0.00 & 0.00 \\
\hline Se omite la presentación de los efectos fijos y temporales.
\end{tabular}

En la segunda etapa, con regresiones de panel no balanceados específicas para cada tipo de propiedad, resultaron las siguientes muestras: 24 empresas controladas por familias (192 observaciones); 23 empresas controladas por ejecutivos (184); y 21 firmas controladas por multinacionales (168). El Cuadro 11 presenta las regresiones para cada medida de diversificación y tipo de controlador. No fue posible lograr una conclusión consistente sobre la relación entre diversificación y la $Q$-Tobin, para ningún tipo de controlador y segmento.

Se encontró que, cuando el controlador es una familia, existe una relación curvilínea entre desempeño y propiedad del controlador -al igual que McConnell \& Servaes (1990)-, donde en un principio la participación del controlador aporta valor a la empresa -lo que es consistente con la hipótesis de alineación de intereses y compromiso familiar-, para luego decrecer a partir de cierto punto, como lo dictaría la hipótesis de expropiación y la empresa como un feudo. En caso de que el controlador sea un grupo de ejecutivos, para una propiedad superior al $65 \%$ se presenta una relación positiva y significativa con la $Q$-Tobin, tal como lo señala la hipótesis de alineación de intereses. Para las multinacionales no se obtuvieron parámetros significativos.

Se destaca que la variable leverage, para los controladores de tipo familiar, fue negativa y significativa, lo que podría señalar que tales empresas presentan conflictos generacionales que podrían conducir a la quiebra o a la disolución de la empresa. De hecho, en Chile, la media de antigüedad de las empresas familiares medianas y grandes es de 31 años, y sólo el $16 \%$ supera los 50 años de vida. ${ }^{17}$ También destaca el signo positivo y significativo que presentó la variable pago de dividendos en los controladores de tipo ejecutivos, lo cual

17 Management en Empresas Familiares, El Diario Financiero. 
puede interpretarse como evidencia para la teoría de señales. Las restantes variables, como rentabilidad y crecimiento, en general conservaron su signo y significancia estadística.

Cuadro 11: P-value consistente de White en paréntesis.

\begin{tabular}{|c|c|c|c|}
\hline Variables* & $\begin{array}{c}\text { Modelo I } \\
\text { a) })^{* *}\end{array}$ & $\begin{array}{c}\text { Modelo I } \\
\text { b) }\end{array}$ & $\begin{array}{c}\text { Modelo I } \\
\text { c) }\end{array}$ \\
\hline NUM-1 a 2 & $\begin{array}{c}0.0638 \\
(0.77)\end{array}$ & $\begin{array}{r}0.0333 \\
(0.77)\end{array}$ & $\begin{array}{c}-0.2608 \\
(0.32)\end{array}$ \\
\hline NUM-3 & $\begin{array}{c}-0.3012 \\
(0.18)\end{array}$ & $\begin{array}{l}0.3439 \\
(0.01)\end{array}$ & $\begin{array}{l}1.8390 \\
(0.05)\end{array}$ \\
\hline NUM-+3 & $\begin{array}{c}-0.0831 \\
(0.67)\end{array}$ & $\begin{array}{c}-0.2985 \\
(0.10)\end{array}$ & $\begin{array}{c}-0.3920 \\
(0.04)\end{array}$ \\
\hline $\mathrm{H}-0$ a 40 & & & \\
\hline $\mathrm{H}-40$ a 60 & & & \\
\hline $\mathrm{H}-+60$ & & & \\
\hline E-0 a 55 & & & \\
\hline E-55 a 85 & & & \\
\hline $\mathrm{E}-+85$ & & & \\
\hline$\%-0$ a 40 & $\begin{array}{l}8.5338 \\
(0.00)\end{array}$ & $\begin{array}{l}0.6167 \\
(0.73) \\
\end{array}$ & $\begin{array}{c}-3.6019 \\
(0.15)\end{array}$ \\
\hline$\%-40$ a 65 & $\begin{array}{l}0.1585 \\
(0.87) \\
\end{array}$ & $\begin{array}{c}-0.5763 \\
(0.48)\end{array}$ & $\begin{array}{l}1.0255 \\
(0.39) \\
\end{array}$ \\
\hline$\%-+65$ & $\begin{array}{c}-1.6977 \\
(0.04) \\
\end{array}$ & $\begin{array}{l}3.2592 \\
(0.06) \\
\end{array}$ & $\begin{array}{l}0.0634 \\
(0.92) \\
\end{array}$ \\
\hline $\mathrm{A}-0$ a 20 & $\begin{array}{c}-0.4250 \\
(0.00) \\
\end{array}$ & $\begin{array}{c}-0.4126 \\
(0.01) \\
\end{array}$ & $\begin{array}{c}-0.9888 \\
(0.01) \\
\end{array}$ \\
\hline $\mathrm{A}-20$ a 21.5 & $\begin{array}{c}-0.01746 \\
(0.54)\end{array}$ & $\begin{array}{c}-0.0497 \\
(0.74)\end{array}$ & $\begin{array}{c}-0.0931 \\
(0.75)\end{array}$ \\
\hline $\mathrm{A}-+21.5$ & $\begin{array}{c}-0.0966 \\
(0.74) \\
\end{array}$ & $\begin{array}{c}-0.0974 \\
(0.54) \\
\end{array}$ & $\begin{array}{l}2.0152 \\
(0.12) \\
\end{array}$ \\
\hline RO/VTAS & $\begin{array}{l}1.0706 \\
(0.05) \\
\end{array}$ & $\begin{array}{r}0.5329 \\
(0.26) \\
\end{array}$ & $\begin{array}{l}1.1967 \\
(0.03) \\
\end{array}$ \\
\hline $\mathrm{GK} / \mathrm{ACT}$ & $\begin{array}{l}2.3038 \\
(0.01) \\
\end{array}$ & $\begin{array}{l}0.4615 \\
(0.21) \\
\end{array}$ & $\begin{array}{l}0.3299 \\
(0.75) \\
\end{array}$ \\
\hline DIV/ACT & $\begin{array}{c}-0.0534 \\
(0.91)\end{array}$ & $\begin{array}{l}3.2307 \\
(0.00) \\
\end{array}$ & $\begin{array}{l}0.9678 \\
(0.49) \\
\end{array}$ \\
\hline B LP/ACT & $\begin{array}{c}-1.9648 \\
(0.01) \\
\end{array}$ & $\begin{array}{c}-0.7401 \\
(0.16) \\
\end{array}$ & $\begin{array}{l}0.7594 \\
(0.13) \\
\end{array}$ \\
\hline Industria & $\begin{array}{l}0.5143 \\
(0.61) \\
\end{array}$ & $\begin{array}{c}2.5232 \\
(0.00)\end{array}$ & $\begin{array}{l}3.8959 \\
(0.05)\end{array}$ \\
\hline$R^{2}$ Ajustado & $70.7 \%$ & $81.9 \%$ & $67.8 \%$ \\
\hline F-statistic & 28.29 & 39.75 & 16.78 \\
\hline $\mathrm{P}(\mathrm{F}$-statistic $)$ & 0.00 & 0.00 & 0.00 \\
\hline
\end{tabular}


Cuadro 11: (continuación).

\begin{tabular}{|c|c|c|c|}
\hline Variables* & $\begin{array}{l}\text { Modelo II } \\
\text { a) }\end{array}$ & $\begin{array}{l}\text { Modelo II } \\
\text { b) }\end{array}$ & $\begin{array}{l}\text { Modelo II } \\
\text { c) }\end{array}$ \\
\hline \multicolumn{4}{|l|}{ NUM-1 a 2} \\
\hline \multicolumn{4}{|l|}{ NUM-3 } \\
\hline \multicolumn{4}{|l|}{ NUM- +3} \\
\hline $\mathrm{H}-0$ a 40 & $\begin{array}{l}0.6898 \\
(0.17) \\
\end{array}$ & $\begin{array}{c}0.8334 \\
(0.03)\end{array}$ & $\begin{array}{c}-0.7365 \\
(0.35)\end{array}$ \\
\hline $\mathrm{H}-40$ a 60 & $\begin{array}{l}1.1259 \\
(0.34) \\
\end{array}$ & $\begin{array}{l}0.4869 \\
(0.72)\end{array}$ & $\begin{array}{c}3.9064 \\
(0.23)\end{array}$ \\
\hline $\mathrm{H}-+60$ & $\begin{array}{c}-4.8170 \\
(0.07)\end{array}$ & $\begin{array}{c}-0.5802 \\
(0.76) \\
\end{array}$ & $\begin{array}{c}-10.1728 \\
(0.11) \\
\end{array}$ \\
\hline \multicolumn{4}{|l|}{$\mathrm{E}-0$ a 55} \\
\hline \multicolumn{4}{|l|}{ E-55 a 85} \\
\hline \multicolumn{4}{|l|}{$\mathrm{E}-+85$} \\
\hline$\%-0$ a 40 & $\begin{array}{l}9.0685 \\
(0.00) \\
\end{array}$ & $\begin{array}{r}0.3606 \\
(0.83) \\
\end{array}$ & $\begin{array}{l}0.4786 \\
(0.83) \\
\end{array}$ \\
\hline$\%-40$ a 65 & $\begin{array}{l}0.0061 \\
(1.00) \\
\end{array}$ & $\begin{array}{c}-0.5600 \\
(0.47) \\
\end{array}$ & $\begin{array}{l}0.2998 \\
(0.83) \\
\end{array}$ \\
\hline$\%-+65$ & $\begin{array}{c}-1.8186 \\
(0.03) \\
\end{array}$ & $\begin{array}{c}3.5236 \\
(0.03) \\
\end{array}$ & $\begin{array}{l}1.2991 \\
(0.20) \\
\end{array}$ \\
\hline $\mathrm{A}-0$ a 20 & $\begin{array}{c}-0.4885 \\
(0.00) \\
\end{array}$ & $\begin{array}{c}-0.3674 \\
(0.01)\end{array}$ & $\begin{array}{c}-1.2935 \\
(0.02) \\
\end{array}$ \\
\hline $\mathrm{A}-20$ a 21.5 & $\begin{array}{c}-0.01130 \\
(0.69) \\
\end{array}$ & $\begin{array}{c}-0.0595 \\
(0.68) \\
\end{array}$ & $\begin{array}{r}0.2630 \\
(0.50) \\
\end{array}$ \\
\hline $\mathrm{A}-+21.5$ & $\begin{array}{c}-0.0184 \\
(0.94) \\
\end{array}$ & $\begin{array}{c}-0.1058 \\
(0.51) \\
\end{array}$ & $\begin{array}{c}-0.8393 \\
(0.22) \\
\end{array}$ \\
\hline RO/VTAS & $\begin{array}{l}1.0354 \\
(0.06) \\
\end{array}$ & $\begin{array}{l}0.5788 \\
(0.33) \\
\end{array}$ & $\begin{array}{l}1.7548 \\
(0.04) \\
\end{array}$ \\
\hline $\mathrm{GK} / \mathrm{ACT}$ & $\begin{array}{r}2.2009 \\
(0.01) \\
\end{array}$ & $\begin{array}{l}0.3071 \\
(0.42) \\
\end{array}$ & $\begin{array}{l}1.6049 \\
(0.12) \\
\end{array}$ \\
\hline $\mathrm{DIV} / \mathrm{ACT}$ & $\begin{array}{c}-0.0311 \\
(0.95) \\
\end{array}$ & $\begin{array}{c}3.3488 \\
(0.00) \\
\end{array}$ & $\begin{array}{l}1.6755 \\
(0.38) \\
\end{array}$ \\
\hline $\mathrm{B} \mathrm{LP} / \mathrm{ACT}$ & $\begin{array}{c}-2.2371 \\
(0.01) \\
\end{array}$ & $\begin{array}{c}-0.6158 \\
(0.27) \\
\end{array}$ & $\begin{array}{l}0.9697 \\
(0.17) \\
\end{array}$ \\
\hline Industria & $\begin{array}{c}0.5566 \\
(0.58) \\
\end{array}$ & $\begin{array}{l}2.4849 \\
(0.00)\end{array}$ & $\begin{array}{l}5.5127 \\
(0.07) \\
\end{array}$ \\
\hline$R^{2}$ Ajustado & $71.0 \%$ & $81.6 \%$ & $58.3 \%$ \\
\hline F-statistic & 28.71 & 38.93 & 11.94 \\
\hline $\mathrm{P}$ (F-statistic $)$ & 0.00 & 0.00 & 0.00 \\
\hline
\end{tabular}


Cuadro 11: (continuación).

\begin{tabular}{|c|c|c|c|}
\hline Variables* & $\begin{array}{c}\text { Modelo III } \\
\text { a) }\end{array}$ & $\begin{array}{c}\text { Modelo III } \\
\text { b) }\end{array}$ & $\begin{array}{c}\text { Modelo III } \\
\text { c) }\end{array}$ \\
\hline \multicolumn{4}{|l|}{ NUM-1 a 2} \\
\hline \multicolumn{4}{|l|}{ NUM-3 } \\
\hline \multicolumn{4}{|l|}{$\mathrm{NUM}-+3$} \\
\hline \multicolumn{4}{|l|}{ H-0 a 40} \\
\hline \multicolumn{4}{|l|}{$\mathrm{H}-40$ a 60} \\
\hline \multicolumn{4}{|l|}{$\mathrm{H}-+60$} \\
\hline E-0 a 55 & $\begin{array}{c}0.3579 \\
(0.33)\end{array}$ & $\begin{array}{c}0.3632 \\
(0.16)\end{array}$ & $\begin{array}{c}-1.6832 \\
(0.05)\end{array}$ \\
\hline $\mathrm{E}-55$ a 85 & $\begin{array}{c}0.7640 \\
(0.21) \\
\end{array}$ & $\begin{array}{l}1.1667 \\
(0.04) \\
\end{array}$ & $\begin{array}{c}11.0940 \\
(0.05)\end{array}$ \\
\hline $\mathrm{E}-+85$ & $\begin{array}{c}-1.3281 \\
(0.08)\end{array}$ & $\begin{array}{c}-0.4219 \\
(0.47)\end{array}$ & $\begin{array}{c}-1.3428 \\
(0.25)\end{array}$ \\
\hline$\%-0$ a 40 & $\begin{array}{c}9.0051 \\
(0.00) \\
\end{array}$ & $\begin{array}{c}0.3164 \\
(0.85) \\
\end{array}$ & $\begin{array}{c}0.5796 \\
(0.78) \\
\end{array}$ \\
\hline$\%-40$ a 65 & $\begin{array}{c}-0.0269 \\
(0.98) \\
\end{array}$ & $\begin{array}{c}-0.5942 \\
(0.45) \\
\end{array}$ & $\begin{array}{r}0.7307 \\
(0.58) \\
\end{array}$ \\
\hline$\%-+65$ & $\begin{array}{c}-1.7562 \\
(0.03)\end{array}$ & $\begin{array}{c}3.4024 \\
(0.04)\end{array}$ & $\begin{array}{l}0.5963 \\
(0.40)\end{array}$ \\
\hline A-0 a 20 & $\begin{array}{c}-0.4827 \\
(0.00) \\
\end{array}$ & $\begin{array}{c}-0.3854 \\
(0.01) \\
\end{array}$ & $\begin{array}{c}-0.5660 \\
(0.07)\end{array}$ \\
\hline A-20 a 21.5 & $\begin{array}{c}-0.0779 \\
(0.78) \\
\end{array}$ & $\begin{array}{l}0.0107 \\
(0.94)\end{array}$ & $\begin{array}{c}-0.8173 \\
(0.14) \\
\end{array}$ \\
\hline $\mathrm{A}-+21.5$ & $\begin{array}{c}-0.4482 \\
(0.22) \\
\end{array}$ & $\begin{array}{c}-0.1288 \\
(0.46)\end{array}$ & $\begin{array}{c}-1.4394 \\
(0.08) \\
\end{array}$ \\
\hline $\mathrm{RO} / \mathrm{VTAS}$ & $\begin{array}{l}1.0550 \\
(0.05)\end{array}$ & $\begin{array}{l}0.7687 \\
(0.14)\end{array}$ & $\begin{array}{l}1.1188 \\
(0.05)\end{array}$ \\
\hline $\mathrm{GK} / \mathrm{ACT}$ & $\begin{array}{c}2.2572 \\
(0.01)\end{array}$ & $\begin{array}{c}0.2785 \\
(0.46)\end{array}$ & $\begin{array}{c}0.8485 \\
(0.39) \\
\end{array}$ \\
\hline DIV/ACT & $\begin{array}{c}-0.0498 \\
(0.91)\end{array}$ & $\begin{array}{l}3.1941 \\
(0.00) \\
\end{array}$ & $\begin{array}{l}1.4278 \\
(0.36) \\
\end{array}$ \\
\hline $\mathrm{B} \mathrm{LP} / \mathrm{ACT}$ & $\begin{array}{c}-2.2223 \\
(0.01)\end{array}$ & $\begin{array}{c}-0.6907 \\
(0.20)\end{array}$ & $\begin{array}{l}0.3284 \\
(0.45)\end{array}$ \\
\hline Industria & $\begin{array}{l}0.5580 \\
(0.58)\end{array}$ & $\begin{array}{l}2.4794 \\
(0.00)\end{array}$ & $\begin{array}{l}4.3379 \\
(0.04) \\
\end{array}$ \\
\hline$R^{2}$ A justado & $70.9 \%$ & $81.9 \%$ & $65.5 \%$ \\
\hline F-statistic & 28.55 & 39.83 & 15.34 \\
\hline $\mathrm{P}($ F-statistic $)$ & 0.00 & 0.00 & 0.00 \\
\hline
\end{tabular}

* Se omite la presentación de los efectos fijos y temporales.

** a) $=$ Familia, b) =Ejecutivos, c) = Multinacional. 


\section{Conclusiones}

Este artículo analiza la relación existente entre el desempeño de las empresas chilenas, la diversificación de sus negocios, el grado de concentración de la propiedad y el tipo de controlador. De las hipótesis relacionadas al nivel de diversificación de la empresa, no fue posible lograr una posición concluyente al darse resultados no significativos u opuestos entre los distintos indicadores. En relación con la propiedad del controlador, cuando ésta es mayor al $40 \%$, se observa un efecto positivo y significativo para la diversificación -medida por número de negocios, Herfindahl y Entropía-, concordando con la hipótesis de alineación de intereses. Se destaca que las empresas diversificadas tienden a otorgar un mayor monto en créditos a sus relacionadas en comparación a las focalizadas. Por otro lado, se aprecia que existe una mayor liquidez en las acciones de las empresas diversificadas, lo que está en línea con la mayor dispersión de propiedad y el menor porcentaje mantenido por el accionista controlador. También se encuentra una débil evidencia a favor de la postura de que los flujos internos de la empresa constituyen un instrumento que permite expropiar riqueza a los minoritarios, antes que reflejar un mercado de capital interno eficiente. Sobre el impacto que tiene la concentración de la propiedad en el desempeño de la empresa, los resultados de mayor relevancia son alcanzados cuando se hace la distinción por tipo de controlador. Para el caso de las familias, en principio se incrementa el desempeño de la empresa hasta una participación del $40 \%$, para luego caer a partir de un porcentaje de propiedad del $65 \%$. Para el caso de los ejecutivos, la evidencia mostró que su participación no tiene impacto sino hasta que logran una participación del $65 \%$, punto a partir del cual impacta favorablemente el desempeño de la empresa. Por último, en las multinacionales no fue posible distinguir ningún patrón de comportamiento que fuera significativo.

Anexo A.

Listado de empresas en la muestra.

\begin{tabular}{|c|c|c|c|}
\hline $\mathrm{N}^{o}$ & NEMO BOLSA & NOMBRE EMPRESA & $\begin{array}{c}\text { ADMINISTRACIÓN } \\
\text { AÑO 2002 }\end{array}$ \\
\hline 1 & AGUAS & AGUAS ANDINAS S.A. & MULTINACIONAL \\
\hline 2 & ANDINA & $\begin{array}{c}\text { EMBOTELLADORA } \\
\text { ANDINA S.A. }\end{array}$ & FAMILIAR \\
\hline 3 & BESALCO & BESALCO S.A. & FAMILIAR \\
\hline 4 & CAP & CAP S.A. & EJECUTIVOS \\
\hline 5 & CAROLINA & $\begin{array}{c}\text { EMPRESAS SANTA } \\
\text { CAROLINA S.A. }\end{array}$ & FAMILIAR \\
\hline 6 & CCT & $\begin{array}{c}\text { COMPANIA CHILENA } \\
\text { DE TABACOS S.A. }\end{array}$ & MULTINACIONAL \\
\hline 7 & CEMENTOS & CEMENTOS BIO-BIO S.A. & FAMILIAR \\
\hline 8 & CERVEZAS & $\begin{array}{c}\text { COMPANIA CERVECERIAS } \\
\text { UNIDAS S.A. }\end{array}$ & FAMILIAR \\
\hline 9 & CGE & $\begin{array}{c}\text { COMPANIA GENERAL DE } \\
\text { ELECTRICIDAD S.A. }\end{array}$ & EJECUTIVOS \\
\hline
\end{tabular}


(continuación).

\begin{tabular}{|c|c|c|c|}
\hline $\mathrm{N}^{o}$ & NEMO BOLSA & NOMBRE EMPRESA & $\begin{array}{c}\text { ADMINISTRACIÓN } \\
\text { AÑO } 2002\end{array}$ \\
\hline 10 & CHILECTRA & CHILECTRA S.A. & MULTINACIONAL \\
\hline 11 & CHOLGUAN & FORESTAL CHOLGUAN S.A. & FAMILIAR \\
\hline 12 & CINTAC & CINTAC S.A. & EJECUTIVOS \\
\hline 13 & CMPC & EMPRESAS CMPC S.A. & FAMILIAR \\
\hline 14 & COLBUN & COLBUN S.A. & MULTINACIONAL \\
\hline 15 & COLOSO & $\begin{array}{l}\text { SOCIEDAD PESQUERA } \\
\text { COLOSO S.A. }\end{array}$ & FAMILIAR \\
\hline 16 & CONCHATORO & $\begin{array}{l}\text { VINA CONCHA } \\
\text { Y TORO S.A. }\end{array}$ & FAMILIAR \\
\hline 17 & COPEC & $\begin{array}{c}\text { COMPANIA DE PETROLEOS } \\
\text { DE CHILE S.A. }\end{array}$ & FAMILIAR \\
\hline 18 & CRISTALES & $\begin{array}{l}\text { CRISTALERIAS DE } \\
\text { CHILE S.A. }\end{array}$ & FAMILIAR \\
\hline 19 & CRUZBLANCA & ING SALUD S.A. (INGSALUD) & MULTINACIONAL \\
\hline 20 & CTC & $\begin{array}{l}\text { CLA. DE TELECOMUNICA- } \\
\text { CIONES DE CHILE }\end{array}$ & MULTINACIONAL \\
\hline 21 & CTI & $\begin{array}{c}\text { CTI COMPANIA TECNO } \\
\text { INDUSTRIAL S.A. }\end{array}$ & EJECUTIVOS \\
\hline 22 & EDELNOR & $\begin{array}{l}\text { EMPRESA ELECTRICA DEL } \\
\text { NORTE GRANDE S.A. }\end{array}$ & MULTINACIONAL \\
\hline 23 & ELECDA & $\begin{array}{c}\text { EMPRESA ELECTRICA DE } \\
\text { ANTOFAGASTA S.A. }\end{array}$ & MUITINACIONAL \\
\hline 24 & EMELARI & $\begin{array}{c}\text { EMPRESA ELECTRICA } \\
\text { DE ARICA S.A. }\end{array}$ & MULTINACIONAL \\
\hline 25 & ENAEX & ENAEX S.A. & EJECUTIVOS \\
\hline 26 & ENDESA & $\begin{array}{c}\text { EMPRESA NACIONAL DE } \\
\text { ELECTRICIDAD S.A. }\end{array}$ & MULTINACIONAL \\
\hline 27 & ENERSIS & ENERSIS S.A. & MULTINACIONAL \\
\hline 28 & ENTEL & $\begin{array}{l}\text { EMP. NACIONAL DE TELE- } \\
\text { COMUNICACIONES S.A. }\end{array}$ & MULTINACIONAL \\
\hline 29 & EPERVA & $\begin{array}{c}\text { EMPRESA PESQUERA } \\
\text { EPERVA S.A. }\end{array}$ & FAMILIAR \\
\hline 30 & ESVAL & $\begin{array}{c}\text { EMP. OBRAS SANITARIAS } \\
\text { VALPARAISO S.A. }\end{array}$ & MULTINACIONAL \\
\hline 31 & GASCO & GASCO S.A. & EJECUTIVOS \\
\hline 32 & GENER & AES GENER S.A. & MULTINACIONAL \\
\hline 33 & IANSA & EMPRESAS IANSA S.A. & MULTINACIONAL \\
\hline 34 & INFORSA & INDUSTRIA FORESTAL S.A. & FAMILIAR \\
\hline 35 & ITATA & PESQUERA ITATA S. A. & FAMILIAR \\
\hline 36 & LAN & LAN CHILE S.A. & EJECUTIVOS \\
\hline
\end{tabular}


Jiménez, G. (2001). Ethos and Corporate Strategies of Business Groups in Emerging Markets: The Case of Chile. Universidad Adolfo Ibáñez, Documento de Trabajo $N^{\circ} 22$.

Khanna, T. and P. Krishna (1999). Policy Shocks, Market Intermediaries, and Corporate Strategy: The Evolution of Business Groups in Chile and India. Journal of Economics and Management Strategy, 8(2).

Khanna, T. and P. Krishna (1999). Emerging Market Business Groups, Foreign Investors, and Corporate Governance. National Bureau of Economic Research, Working Paper 6955.

Khanna, T. and P. Krishna (1999). Is Group Affiliation Profitable in Emerging Markets? An Analysis of Diversified Indian Business Groups. Harvard Business School.

Lang, L. H. and R. Stulz (1994). Tobin's Q, Corporate Diversification, and Firm Perfomance. Journal of Political Economy, pp. 1248-1280.

La Porta, R., F. Lopez-de-Silanes, A. Shleifer, and R. Vishny (1997). Legal Determinants of External Finance. Journal of Finance, 52, pp. 1131-115.

Lins, K. and H. Servaes (1999). International Evidence on the Value of Corporate Diversification. Journal of Finance, 54, pp. 2215-2239.

Lins, $\mathbf{K}$ and H. Servaes (2002). Is Corporate Diversification Beneficial in Emerging Markets? Financial Management, pp. 5-31.

Majluf, N., N. Abarca, D. Rodríguez, and L. Fuentes (1998). Governance and Ownership Structure in Chilean Economic Groups. Revista ABANTE, 1(1), pp. 111-139.

Mansi, S. and D. Rebb (2002). Corporate Diversification: What Gets Discounted? Journal of Finance, 57, pp. 2167-2183.

Maquieira, C., S. Zurita, V. García, and M. Velasco (2003). Valuation of Investment Companies in Chile. Estudios de Administración, 10(2), pp. 1-25.

McConnell, J. and H. Servaes (1990). Additional Evidence on Equity Ownership and Corporate Value. Journal of Financial Economics, 27, pp. 595-612.

Morck, R., A. Shleifer, and R. Vishny (1998). Management Ownership and Market Valuation. Journal of Finance Economics, pp. 293-315.

Stein, J. (1997). Internal Capital Markets and the Competition for Corporate Resources. Journal of Finance, 52, pp. 111-133.

Tarziján, J. (1999). Internal Capital Markets and Multimarket Contact as Explanations for Conglomerates in Emerging Markets. Revista ABANTE, 2(1), pp. 3-22.

Villalonga, B. (2002). Does Diversification cause the Diversification Discount. Harvard Business School, Working Paper 2002. 